\title{
Fiber optical network design problems: A case for Turkey ${ }^{\text {is }}$
}

\author{
Başak Yazar, Okan Arslan, Oya Ekin Karaşan*, Bahar Y. Kara \\ Bilkent University, Department of Industrial Engineering, Bilkent, 06800 Ankara, Turkey
}

\section{A R T I C L E I N F O}

\section{Article history:}

Received 12 December 2014

Accepted 2 October 2015

Available online 8 October 2015

\section{Keywords:}

Location

Allocation

Telecommunications

Application

\begin{abstract}
A B S T R A C T
In this paper, we consider problems originating from one of the largest Internet service providers operating in Turkey. The company mainly faces two different design problems: the green field design (area with no Internet access) and the copper field re-design (area with limited access over copper networks). In the green field design problem, the aim is to design a least cost fiber optical network that will provide high bandwidth Internet access from a given central station to a set of aggregated demand nodes. Such an access can be provided either directly by installing fibers or indirectly by utilizing passive splitters. Insertion loss, bandwidth level and distance limitations should simultaneously be considered in order to provide a least cost design to enable the required service level. In the re-design of the copper field application, the aim is to improve the current service level by augmenting the network with fiber optical wires, specifically by adding cabinets to copper rings in the existing infrastructure and by constructing direct fiber links from cabinets to distant demand nodes. Mathematical models are constructed for both problem specifications. Extensive computational results based on realistic data from Kartal (45 nodes) and Bakırköy (74 nodes) districts in Istanbul show that the proposed models are viable exact solution methodologies for moderate dimensions.
\end{abstract}

(c) 2015 Elsevier Ltd. All rights reserved.

\section{Introduction}

In telecommunications networks, design problems involve either constructing the network from scratch or improving the existing network in terms of capacity or speed. Our problem is motivated from a real-world application of Turkey's largest service provider. In Turkey, due to the competitive environment in the telecommunications market and privatization of big companies, a highly qualified, efficient and cost effective service in data communication is crucial. The practice of the market leader is critical for today's market share and will determine the market positioning in the near future. Following the needs of our service provider, we introduce two different problem definitions to the literature, namely, the green field network design problem and the copper field re-design problem.

In the green field, the fiber optical network is to be designed from scratch. Every customer point will be reachable from a central station. This central station is assumed to have a direct link to upper level networks and reach world-wide Internet. In reaching

\footnotetext{
This manuscript was processed by Associate Editor Lodi.

* Corresponding author. Tel.: +90 312290 1409; fax: +90 3122664054 .

E-mail addresses: basakyazar@gmail.com (B. Yazar),

okan.arslan@bilkent.edu.tr (O. Arslan), karasan@bilkent.edu.tr (O.E. Karaşan), bkara@bilkent.edu.tr (B.Y. Kara).
}

the customers, different numbers and types of passive splitters, which are special telecommunication equipments that split the incoming fiber optical wire into several different wires carrying the same data signal, are utilized. Due to the application dynamics of the problem, the service quality is measured in terms of bandwidth and insertion loss.

In the copper field, the design problem seeks to provide improvement in an area that the company has already been serving via copper cables. The existing copper infrastructure will be augmented with fiber optical wires in order to improve the speed of the Internet at the desired points.

\subsection{Green field network design problem}

The Information and Communication Technologies Authority in Turkey regulates that any new customer should be served with a fiber Internet access so as to ensure either directly or through capacity expansion, the future demand. To this end, the green field design problem is solved in areas where the service provider currently has no infrastructure at all. Aggregated demand locations are to receive high-speed Internet access as defined by the service provider. In the particular setting under consideration, there is a central station at a fixed location. Each customer needs to be connected to this central station via a path of fiber links to be installed. Passive splitters will aid in carrying data in bulk up to 


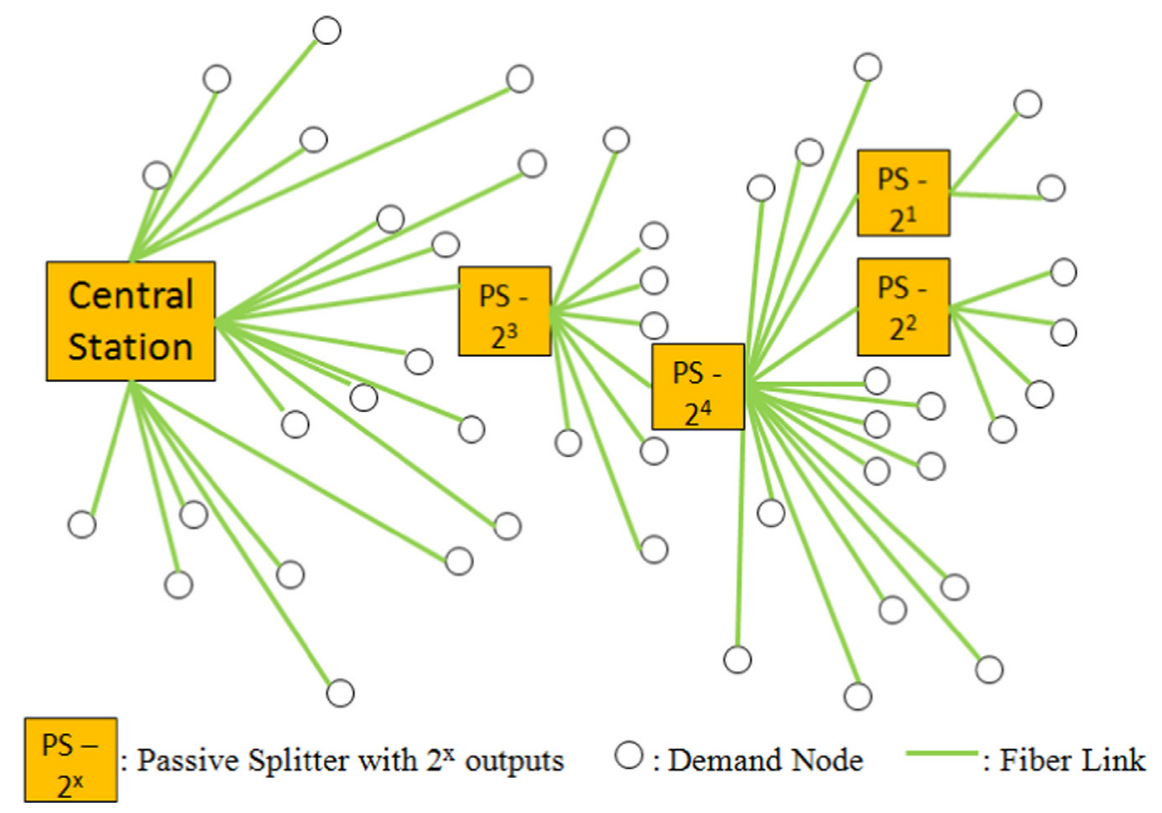

Fig. 1. Green field network design problem illustration.

certain locations to avoid costly direct connections from the central station. Thus, the locations and the splitting capacities of the passive splitters are very crucial in a cost-effective design. Furthermore, such decisions are crucial in the resulting quality of service. Fig. 1 illustrates a sample green field design.

One aspect of the quality of service is measured in terms of decibels $(\mathrm{dB})$. The $\mathrm{dB}$ level of data experiences insertion loss and decays with the distance travelled and with the increasing number of ports encountered at the splitters. The requirement is to have all demand points within a $\mathrm{dB}$ radius from the central station. Another service quality criterion is the speed of the Internet access. Originating from the central station, the bandwidth power splits into the number of ports it encounters at a passive splitter. The service provider would like to serve all its customers with a minimum threshold level of bandwidth.

Decisions entailing to the location and the type of passive splitters involve a trade-off between the cost of fiber cables, the insertion loss and the bandwidth division. In this respect, the locations of the splitters, their capacities, and their allocations should be made considering competing objectives.

In summary, the green field design problem includes the selection of passive splitter locations, their splitting numbers and fiber links between demand nodes and passive splitters including the central station. While minimizing the total costs, the insertion loss requirements and bandwidth target values should be respected.

\subsection{Copper field re-design problem description}

The second problem seeks to improve the existing telecommunications service in a region named as the "copper field". The copper field is composed of some copper or related wires that are used for normal speed data transmission. In order to improve the Internet access bandwidth, fiber wires could be used completely from the central station to the end-user or to a certain point closer to the end-user. Hence, data transmission can be performed with both fiber and copper wires and this hybrid usage of fiber and copper will lead to a quicker, safer and a better performing Internet access. Fiber cables starting from the central station are to be wired up to some point in the network and the remaining transmission is to be done via existing copper cables.
Since data travels a shorter distance on the copper wires, the transmission speeds up.

There is a central station which is already in use for Internet access via copper wires. The existing network consists of rings (loops) of copper cables around the central station. There might be one or more copper cable rings around a central station depending on the geographical span of the region. Fiber wiring should start from the central station and touch the copper ring(s) at some point(s) to improve the bandwidth. Such a point of touch needs a special equipment called "cabinet". The cabinet acts as the central station of its ring and it is assumed that a cabinet provides the same quality of service as a central station does. Each cabinet is required to be connected to the central station via two arc disjoint paths to ensure a reliable connection to the backbone network. This property comes out as a ring structure between cabinets including the central station as depicted in Fig. 2. In this manner, the central station can also be considered as a cabinet.

If any demand node is in close proximity (say at most $\gamma$ meters) to a cabinet over the copper wiring, the particular demand node is accepted as receiving fiber access. Hence, demand nodes in $\gamma$ neighborhood over the copper ring to a cabinet can be covered and served by this cabinet. If a node is located further than this proximity from a cabinet, it is not considered as served by the cabinet, in which case a direct fiber cable from the cabinet to this node is required. Note that, for a node, being geographically close to a cabinet node does not qualify it to be in the $\gamma$ neighborhood of the cabinet. The node and the cabinet also need to be in the same copper ring as well.

In the network design problem over an existing copper network, some customers may require to use fully fiber wire. Such customers are referred to as premise customers by our company. Thus, they require a sole fiber path from the central station up to their building. In addition, premise nodes also require having a direct connection to at least one other premise node. Fig. 2 illustrates such premise customers and their linkage demands.

In the copper field network re-design problem, there is also a distance restriction for the fiber access measured from the central station up to the end-user. This distance is a threshold for wiring the fiber cable.

Our aim is to serve all nodes with fiber connectivity by installing a minimum cost fiber network over the existing one. 


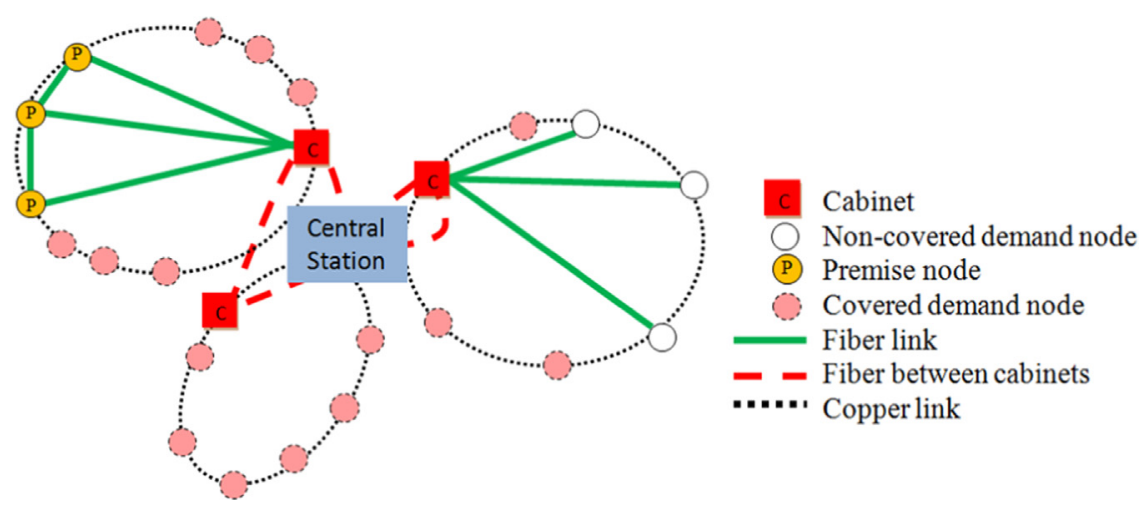

Fig. 2. Illustration of copper field re-design problem.

\section{Related Literature}

For designing the infrastructure of telecommunications networks, many disciplines including operations research, computer science and electrical and electronics engineering examine the problem from their respective perspectives. Within the context of operations research, a telecommunications network consists of a set of nodes and a set of links joining some pairs of nodes [1]. These nodes are demand points that send/receive messages or information such as voice, data, and video. The transmission is done via communication links [1]. The communication links can be copper cables (coaxial cable or twisted pair), fiber ones or both. In the OR literature, the telecommunications network design problem (TNDP) is defined as finding a suitable configuration of nodes and links, so as to satisfy the traffic demand between nodes. In both expansion and designing from scratch cases, the resulting networks have many evaluation criteria. These are network cost, capacity, reliability, performance, and demand pattern [1].

When we look at the backbone/access network differentiation from a hub location perspective, a telecommunications network consists of tributary and backbone networks. Hubs are switching points of the telecommunications traffic. Tributary networks (local access networks) connect demand nodes to hubs, while backbone networks interconnect the hubs. In this definition, tributary networks can be local or access networks and backbone networks can be hub-level networks. Hubs are telecommunication elements such as switches, gates, concentrators, control points, or access points [1]. In general, the multi-level network infrastructures include multiple levels that are connected to each other in a hierarchical manner. There are different types of problems that consider (i) solely access or (ii) solely backbone networks as well as (iii) joint problems which evaluate backbone and access networks together. Since the telecommunications network problems are in general very challenging, a typical research divides the core problem into subproblems and solves them subsequentially [2,3]. The subproblems are generally NP-hard [4]. The local access network design problem could be concentrator location problem, terminal assignment problem, terminal layout problem or Telpak problem [2-10]. For further information about re-design and update problems in the local access networks, the reader can refer to surveys [11-14]. For earlier works, the reader can refer to $[3,10,15,16]$. In the backbone network design problem, different types of solution techniques including heuristics, such as branch exchange, concave branch elimination or cut saturation are used [17-19]. More general surveys about the joint problems include [20-29].

In a fiber optical access network, an acronym FTTX (Fiber-tothe- $\mathrm{X}$ ) is used for identification depending on the fiber length. If the fiber ends in a street cabinet, it is called fiber-to-the-node or neighborhood (FTTN). On the other hand, if the cabinet is closer to the user's premises, this is fiber-to-the-cabinet or curb (FTTC). Fiber-to-the-building, -business, -basement (FTTB) is used for fiber connection up to the basement of a building. Full fiber Internet access exists in the fiber-to-the-home (FTTH) and fiber-to-thedesktop [30].

Recall that when designing the network from scratch in the green field, the goal is to reach every customer in a cost effective way by utilizing passive splitters. The passive splitters are generally used in physical networks such as Ethernet passive optical networks and wavelength division multiplexing passive optical networks for FTTH cases [31]. In the passive optical networks (PONs), all elements starting from supplier, which is the optical line terminal, to the customer, which is the optical network terminal, are passive. From a central office/station, there is a feeder section including an optical line terminal and an optical distribution point. Then, the distribution is handled via a splitter and optical wire is reached first to the optical terminal panel, then to the optical network terminal at the customer site [32]. In FTTH case, Kim et al. [32] propose a PON formulated as a multi-level (primary and secondary) capacitated facility location problem with non-linear link costs. Similarly, Brittian et al. [33] use a formulation by fixing the primary nodes and optimizing the secondary ones depending on the split levels at the primary nodes and then making an allocation of the customers to the secondary nodes. The aim is the minimization of the network design cost. Another multilevel optical FTTH network design model with given edge capacities is proposed and proved as an NP-hard problem in [34]. A heuristic based PON network planning is proposed in [35]. A metropolitan area PON design in an application based problem tested in Turkey is formulated in [36].

Many different studies are considered in designing a PON. These include choosing the topology, location, allocation and capacity planning [37]. The green field network design problem has a multicommodity flow pattern. Since passive splitters have a limited outgoing fiber number, the problem is capacitated. Similarly, different passive splitter types show the multi-facility property of the problem. A tree of passive splitters rooted at the central station is to be designed. All the demand nodes will be allocated to either the central station or to one of the located passive splitters. Since we do not know the number of levels in the embedded tree network in advance, the problem definition differs from the ones in the literature [37]. There is no explicit bound for the number of splitters on the unique path from the central station to a demand point. The quality of service requirements in terms of speed and $\mathrm{dB}$ radius is the only determining factors in the resulting multi-level infrastructure of the green design, a main point of divergence from the literature. We refer the interested reader to [38] for PON architecture and operation principles. Lagrangian based mathematical formulations are used for bi-level FTTx PON structures in [39]. 
More recently, the tree of hubs location problem has been introduced [40]. Its particularity is that the hubs are required to be connected by means of a tree. Those nodes that are not on the tree of hubs are assigned to one of the hub nodes on the tree to receive service. In this respect, we can view the tree of hubs problem as a joint modeling technique similar to ours to design both tributary and backbone networks. The heterogeneity of the hubs and the quality of service requirements make our green field network design problem more challenging than the tree of hubs design problem. Different models [41] and solution techniques such as Benders Decomposition [42] are also present in the recent literature.

Different variants of the two-level network design problems exist in the literature. An Ethernet-based local area access network design problem with end-to-end quality-of-service (QoS) constraints is proposed in [43]. The problem is to design a two-level hierarchical network on the tree topology. Different than other studies, the authors consider end-to-end QoS requirement which is defined as a nonlinear function of the blocking probability of access and aggregation switches. Another variant of this problem is applied in Seoul metro area [44]. Two-level network design problem is also applied to the electrical distribution system [45]. In their particular application, the facilities are partitioned into two sets and there are additional constraints regarding these sets.

Studies tuned to the particular needs of telecommunications company's problems view telecommunications network planning as a strategic level decision [46-49]. In this study, we also adopt a similar viewpoint. Our copper field re-design problem is about an improvement in the existing network. The infrastructure of a given network consists of copper rings that are connected to the central station. In the re-design model we determine the location of cabinets in these copper rings and connect the cabinets with central station in a ring structure. Also, additional fibers are installed depending on the coverage threshold value prescribed by $\gamma$. Besides these fiber links, the premise nodes are linked to their closest premise neighbors. The remaining characteristics include multi-commodity flow pattern and single facility type since all cabinets are homogeneous.

Within the scope of this study, application dynamics derived for both problems are embedded in the proposed mathematical models. The individualized problem requirements differentiate our problems and our models from the ones proposed in the literature.

\section{Model development}

\subsection{Green field model development}

In the green field network design, given a set of demand locations, we need to find passive splitter locations to reduce direct fiber optical wire usage. Without loss of generality, we assume that all demand points are candidate passive splitter locations. Let $N$ be the demand node set on a network including a central station which is referred to as central.

Different passive splitter types are considered in the system. Let $T$ be the set of passive splitters' types and $L=\left[l_{i j}\right], i, j \in N$ be the highway distance from node $i$ to node $j$. Since we aim to minimize the cost, we include the fiber optical wire cost in the function. $C=\left[c_{i j}\right], i, j \in N$ represents the cost of installing a fiber link from $i$ to $j$. Let $s_{k}$ be the cost of $k$ th type passive splitter where $k \in T$. With the current technology, the passive splitter cost is far dominated by the cost of installing fiber links in real world [50], which is also the case in our particular application setting. However, as technological developments increase and the specs improve, the relative cost of passive splitters and fiber cables may vary. To this end, we define a parameter $\alpha$ corresponding to the proportion between the splitter and wiring costs. An alpha value of 1 implies that $1 \mathrm{~km}$ of fiber cable can be traded for a splitter with a cost of 1 unit. This proportion allows us to make a comparison for different cost structures of the fiber optical wiring and passive splitters and to draw conclusions about the computational challenges of the green field network design problem. We also denote the port number in the splitter by parameter $f_{k}, k \in T$.

The insertion loss in each level of port is specified with the parameter declineps. Similarly, the insertion loss per kilometer is another power loss in the system, defined by the parameter declineway in our model. dBcapacity defines the threshold loss value; in other words, it is the total insertion loss budget. Without loss of generality, we assume that a signal starts at the central station with zero $\mathrm{dB}$ and each fiber optical wire length traversed augments the $\mathrm{dB}$ amount. Also, passive splitters contribute to that amount. Thus, every node needs to be within this dBcapacity radius.

The service quality of the company is measured by the download speed in units of $\mathrm{MB} / \mathrm{s}$. The out power of the central station is determined by the parameter mbcentral. The aim of the service provider is to provide at least mbthreshold MBs, to represent the download speed threshold for each distribution point. This specification is also affected by the number of ports in the passive splitters. More splitting causes more reduction in the speed.

Decision variables are as follows:

$x_{\mathrm{ij}}=\left\{\begin{array}{ll}1 & \text { if } \operatorname{arc}(i, j) \text { is on the tree } \\ 0 & \text { otherwise, }\end{array} \quad i, j \in N\right.$

$z_{\mathrm{ij}}=\left\{\begin{array}{ll}1 & \text { if } i \text { is assigned to } j ; z_{\mathrm{ii}} \text { will be } 1 \text { if } i \text { is a hub node } \\ 0 & \text { otherwise, }\end{array} \quad i, j \in N\right.$

$y_{\mathrm{jk}}=\left\{\begin{array}{ll}1 & \text { if } k^{\text {th }} \text { type of passive splitter is located at node } j \\ 0 & \text { otherwise, }\end{array} \quad k \in T, j \in N\right.$

$m_{j}=$ the out speed at a node $j \in N$ in terms of $\mathrm{MB} / \mathrm{s}$

$p_{j}=$ the $\mathrm{dB}$ amount at node $j \in N$

We call the solution of the green field design problem as a network, and the arcs in this network as 'network arcs'. A network is composed of two different types of arcs. One type lies on the tree of hubs rooted at central, which is defined by $x_{i j}$ variables. Therefore, we call $x$ as tree variables, and those arcs on the rooted tree as tree arcs. The second type of arcs appear between leaf nodes and the hub nodes and are indexed by the $z_{i j}$ variables. Following the tradition in the hub location literature, we call the $z$ variables as assignment variables, and those arcs as assignment arcs. Apart from assigning leaf nodes to hub nodes, the $z_{i j}$ variables also declare a node as hub, if $i=j$.

The green field network design model is

$\min \sum_{i \in N j \in N: j \neq i} \sum_{i j} x_{i j}+\sum_{i \in N j \in N: j \neq i} \sum_{j i} z_{i j}+\sum_{i \in N k \in T} \sum_{k} \alpha s_{k} y_{i k}$

subject to

$$
\begin{array}{r}
\sum_{j \in N: j \neq i} x_{j i}=z_{i i} \quad \forall i \in N \backslash \text { central } \\
z_{i i} \leq \sum_{j \in N: j \neq i} x_{i j}+\sum_{j \in N: j \neq i} z_{j i} \quad \forall i \in N
\end{array}
$$

$\sum_{j \in N: j \neq i, \text { central }} x_{i j}+\sum_{j \in N: j \neq i, \text { central }} z_{j i} \leq \sum_{k \in T} 2^{f_{k}} y_{i k} \quad \forall i \in N \backslash$ central

$\sum_{j \in N} z_{i j}=1 \quad \forall i \in N$ 


$$
\begin{aligned}
& \sum_{k \in T} y_{j k}=z_{j j} \quad \forall j \in N \backslash \text { central } \\
& z_{\text {central,central }}=1 \\
& p_{j} \geq p_{i}+\text { declineway } l_{i j} x_{i j}+\sum_{k \in T} \text { declineps }_{k} y_{j k}-M\left(1-x_{i j}\right) \\
& \quad \forall i, j \in N, \quad i \neq j, j \neq \text { central } \\
& p_{i}+\text { declinewayl } z_{i j} z_{j i} \leq d B c a p a c i t y \quad \forall i, j \in N, i \neq j \\
& m_{\text {central }}=\text { mbcentral } \\
& m_{j} \leq \frac{m_{i}}{2^{f_{k}}}+m b c e n t r a l\left(1-y_{j k}\right)+m b c e n t r a l\left(1-x_{i j}\right) \\
& \quad \forall k \in T, i, j \in N, i \neq j, j \neq \text { central } \\
& m_{j} \geq m b t h r e s h o l d z_{j j} \quad \forall j \in N \backslash \text { central } \\
& x_{i j}, z_{i j}, y_{j k} \in\{0,1\} \quad \forall i, j \in N, k \in T \\
& p_{j}, m_{j} \geq 0 \quad \forall j \in N
\end{aligned}
$$

The objective function (1) minimizes the total cost of passive splitters and the fiber optic cables. The first term in the objective function is related to the tree arcs, the second term is for the assignment arcs and the last term is for the cost of passive splitters. Constraints (2) ensure that if a node is a hub, then there must be an incoming tree arc. Conversely, if there is an incoming tree arc to a node, then that node is allocated as a hub. Constraints (3) have a similar logic. If a node is a hub, then a network arc must leave this node. Constraints (4) limit the outgoing arcs of a hub node according to the splitter type located at that node. Constraints (5) either assign a node to a hub or declare that node itself as a hub. Therefore, we make sure that every node receives service. Constraints (6) ensure that one type of passive splitter is assigned to a hub node. Constraints (7) declare the central node as a hub. But since Constraints (2), (4) and (6) exclude central nodes, the capacity and incoming arc limitations of a hub node do not hold for the central node.

To enforce the insertion loss requirements, Constraints (8) and (9) are used. With Constraints (8), each node's dB amount is calculated based on its unique path from the central node. As a convention, we calculate the $\mathrm{dB}$ requirement beginning from the central station and the central station has $0 \mathrm{~dB}$ value. The $\mathrm{dB}$ values of all nodes need to be within the insertion loss budget. If there is a link between two nodes, then the $\mathrm{dB}$ amount increases from the previous one according to the length of the fiber. Also, if the specific node is selected as a passive splitter location, it increases the $\mathrm{dB}$ amount depending on its type. Constraint (9) ensures that every node's $\mathrm{dB}$ amount is within the $\mathrm{dB}$ insertion loss budget. These two constraints also prevent subtours. In the bandwidth calculation, the central station begins with the mbcentral parameter value (Constraint (10)) and decreases through the splitters. With Constraints (11), if there is a tree arc from splitter $i$ to splitter $j$, then splitter $j$ 's out dB level decreases from $i$ 's out $\mathrm{dB}$ level depending on its splitter type. The limitations of $\mathrm{dB}$ amounts are specified with Constraints (12). Finally, constraints (13) and (14) are the domain restrictions. (14):

The followings are valid inequalities/optimality cuts for (2)-

$$
\begin{aligned}
& \sum_{i \in N j \in N: j \neq i} \sum_{i j} x_{j \in N} z_{j j}-1 \\
& \sum_{i \in N j \in N: j \neq i} \sum_{i j} x_{i \in N j \in N: j \neq i} \sum_{i j} z_{i j}=|N|-1 \\
& m_{i} \leq \text { mbcentralz } i i \quad \forall i \in N
\end{aligned}
$$

$$
\begin{aligned}
& p_{j} \leq d \text { Bcapacityz } j j \quad \quad \forall j \in N \\
& x_{i j}+x_{j i}+z_{i j}+z_{j i} \leq 1 \quad \forall i, j \in N: i \neq j \\
& z_{i j}+x_{i j}+x_{j i} \leq z_{j j} \quad \forall i, j \in N: i \neq j
\end{aligned}
$$

Constraints (15) simply state that the number of arcs is equal to the number of hubs less one. Similar to tree of hubs, the designed network itself is also a spanning tree. Therefore, the sum of the tree variables and the assignment variables equals $|N|-1$ (Constraints (16)). Since $m$ variables keep track of outgoing MB values at the splitters, a non-hub node can be assigned an $m$ value of zero, an optimality cut enforced by Constraints (17). Constraints (18) enforce the equivalent conditions of Constraints (17) for $\mathrm{dB}$ values. Constraints (19) state that an arc can only be either a tree arc or an assignment arc in one direction. Lastly, Constraints (20) state that if there is a tree arc incident to a node $j$, or another node $i$ is assigned to node $j$, then node $j$ is necessarily a hub node.

\subsection{Copper field re-design model development}

A mixed integer formulation using the same notation set $N$, central and $L=\left[l_{i j}\right]$ as in the green field model formulation, is proposed. The distance capacity from the central station till the demand node is considered in this model, and the maximum distance is expressed with $H$. The number of cabinets is fixed to parameter $p$. Since cabinets have degree two, the path length at each cabinet from the central station should be calculated both clockwise and counterclockwise. For the rest of the demand nodes, the path lengths from the central station should be considered as the summation of the largest path length at the cabinet and the distance between the cabinet and that particular node. Therefore, we evaluate the path length at any cabinet from two directions (clockwise/counterclockwise) and consider the largest one for the rest of the calculations.

A covered node means that a cabinet is close enough to it, so the node has an Internet service better than before but not necessarily as fast as fully fiber optical Internet access. For this purpose, for each node $i \in N$ we define a set $P L_{i}$ which holds potential cabinet locations which can cover node $i$ within $\gamma$ value along copper cable, i.e., $P L_{i}=\left\{j \in N: d_{i j} \leq \gamma\right\}$. Observe here that a cabinet can cover a node only if the node is in its copper ring. Thus, we need another distance matrix $D=\left[d_{i j}\right]$, which represents the distances between all nodes on the same copper ring, i.e., if two nodes, say $a$ and $b$, are on different copper rings, $d_{a b}$ gives an infinite value.

Set $P$ is the given set of premise nodes. A premise node is to be connected to its closest neighboring premise node. We assume that a single premise node has two terminal points that fiber wire can enter and leave, and without loss of generality it is assumed that these are two different demand nodes. Therefore, there cannot be a single excluded/isolated node in the area. In order to connect to the nearest premise node, we need to keep the closest premise neighbor for each premise node. For every $i \in P$, we let $R(i)=\operatorname{argmin}\left\{l_{i j}: j \in P, j \neq i\right\}$.

Decision variables are as follows:

$w_{i j}= \begin{cases}1 & \text { if we build a fiber wire between two cabinets } i \text { and } j ; i \neq j, i, j \in N \\ 0 & \text { otherwise, }\end{cases}$ $x_{i j}= \begin{cases}1 & \text { if there is fiber link from cabinet } i \text { to node } j ; i \neq j, i, j \in N \\ 0 & \text { otherwise, }\end{cases}$ $z_{j}= \begin{cases}1 & \text { if demand node } j \text { is selected as cabinet } j \in N \\ 0 & \text { otherwise, }\end{cases}$ $y_{j}= \begin{cases}1 & \text { if demand node } j \text { is served by a cabinet via copper wire; } j \in N \\ 0 & \text { otherwise, }\end{cases}$ 
$u_{i}=$ the path length from the central to a cabinet located at node $i \in N$

$\bar{u}_{i}=$ the reverse path length at node $i \in N$

$v_{i}=$ the longest path length at node $i \in N$, i.e. $\max \left(u_{i}, \bar{u}_{i}\right)$

$q_{i}=$ the path length from the central to a non-cabinet node located at node $i \in N$

The mathematical model is as follows:

$\sum_{i \in P} \frac{1}{2} l_{i, R(i)}+\min \sum_{i \in N j \in N: j \neq i} \sum_{i j}\left(x_{i j}+w_{i j}\right)$

subject to

$z_{i}+y_{i}+\sum_{\substack{j \in N \\ j \neq i}} x_{j i} \geq 1 \quad \forall i \in N$

$\sum_{\substack{j \in N \\ j \neq i}} w_{i j}+\sum_{\substack{j \in N \\ j \neq i}} w_{j i} \geq 2 z_{i} \quad \forall i \in N$

$\sum_{j \in P L_{i}} z_{j} \geq y_{i} \quad \forall i \in N$

$z_{\text {central }}=1$

$\sum_{k \in N} z_{k}=p+1$

$w_{i j}+w_{j i} \leq 1 \quad \forall i, j \in N, i \neq j$

$\sum_{\substack{j \in N \\ j \neq i}} w_{i j}=z_{i} \quad \forall i \in N, i \neq$ central

$\sum_{\substack{j \in N \\ j \neq i}} w_{j i}=z_{i} \quad \forall i \in N, i \neq$ central

$\sum_{\substack{i \in N \\ i \neq \text { central }}} w_{i, \text { central }}=\sum_{\substack{i \in N \\ i \neq \text { central }}} w_{\text {central }, i}$

$\sum_{\substack{j \in N \\ j \neq \text { central }}} x_{\text {central }, j}+\sum_{\substack{j \in N \\ j \neq \text { central }}} x_{j, \text { central }}=0$

$\sum_{\substack{j \in N \\ j \neq i}} x_{i j} \leq M z_{i} \quad \forall i \in N$

$\sum_{\substack{i \in N \\ i \neq j}} x_{i j}=1 \quad \forall j \in P$

$u_{\text {central }}=0$

$u_{j} \geq u_{i}-H\left(1-w_{i j}\right)+w_{i j} l_{i j} \quad \forall i, j \in N, j \neq$ central,$j \neq i$

$\bar{u}_{i} \geq \bar{u}_{j}-H\left(1-w_{i j}\right)+w_{i j} l_{i j} \quad \forall i, j \in N, i \neq$ central,$j \neq i$

$v_{i} \geq \bar{u}_{i} \quad \forall i \in N$

$v_{i} \geq u_{i} \quad \forall i \in N$

$v_{i} \leq H \quad \forall i \in N$

$q_{j} \geq v_{i}-M\left(1-x_{i j}\right)+l_{i j} \quad \forall i, j \in N, j \neq i$

$q_{R(i)}+l_{R(i), i} \leq H \quad \forall i \in P$

$q_{j} \leq H \quad \forall j \in N$ $w_{i j}, x_{i j}, z_{j}, y_{j} \in\{0,1\} \quad \forall i, j \in N, j \neq i$

$u_{j}, \bar{u}_{j}, v_{j}, q_{j} \geq 0 \quad \forall j \in N$

In the copper field re-design problem the aim is to minimize the fiber optical wiring necessary to provide the required service. The first part of the objective function is about the premise nodes. Since each premise node and its nearest premise node are known in advance, this value is added to the objective function as a constant. The division by 2 is to avoid double counting.

The second part of the objective function is related to the fiber decisions of the model. If there is a link between two cabinets or a link from a cabinet to a node, then these are included in the objective function. The demand satisfaction of every node is guaranteed by Constraint (22). The demand satisfaction can be accomplished in three different ways: (i) a cabinet can be located at a demand node, (ii) a node can be covered via copper lines from a cabinet node in $\gamma$ threshold distance, or (iii) there might be a direct fiber link from a cabinet to a demand node.

A cabinet node has degree 2 via Constraint (23). The coverage of demand nodes within $\gamma$ distance is handled via Constraint (24). The central station is assumed to act like a cabinet via Constraint (25). Therefore, the total number of cabinets is equal to the required number of cabinets plus 1 , as in Constraint (26).

Constraints (27)-(29) assign $w$ values only between two cabinets and allow unidirectional fiber usage. There could be more than one ring connected to the central station. Thus, the flow balance constraints for the central station are given in Constraint (30).

We assume that no node can be directly linked to the central station, which is satisfied via Constraint (31), since the central station can only be connected to the other cabinets. If there is no cabinet in node $i$, there cannot be an outgoing link from that particular node (Constraint (32)).

For the premise nodes, there has to be exactly one incoming link from any cabinet and that is enforced with Constraint (33).

In Constraint (34), the path length of the central station is fixed to zero. Through constraints (35) and (36), clockwise and anticlockwise path lengths of cabinets from the central station are calculated. Constraints (37)-(39) bound the maximum of these two distance calculations, namely $v_{i}$, to be at most the threshold value $H$.

For the nodes that are linked to cabinets, the distance from the central station is calculated by using Constraint (40). If this is a premise node, its longest distance from the central station includes the neighboring premise-node-link as well. This is ensured with Constraint (41). The path lengths for demand nodes cannot exceed the distance threshold value as dictated by Constraint (42). The rest of the constraints (43)-(44) are the domain restrictions.

\subsection{Implementation details}

All computational studies are performed on a Linux environment with $4 \times 16$ C AMD opteron with 96 GB RAM using Java and CPLEX 12.5. 10-h time limit is set for all problem instances. In an effort to improve the solution time of the green field design problem, we added all cuts presented in (15)-(20). Observe that we are in search for a hub network design such that a minimum QoS in terms of $\mathrm{dB}$ and $\mathrm{MB}$ is provided at each node. Constraints (2)(7) are related to network design while Constraints (8)-(12) maintain the service level as desired. Especially this second set of constraints complicates the solution process due to the MillerTucker-Zemlin [52] nature of (8) and (11). Therefore, we tested adding these constraints as lazyconstraints in Cplex. This effectively turns the problem into a branch and cut framework in which an 
integer solution satisfying the first set of constraints is searched for. Once found, the lazy constraints are controlled for possible violation. If that is the case, the violated constraints are added to the problem. Otherwise, if all the lazy constraints are also satisfied, then the integer solution is accepted as the incumbent. However, as an experience, this did not provide overall runtime improvement. On the other hand, we gained runtime improvement by changing the settings of Cplex to aggressively generate cuts in the root node of the branch and bound tree in both problem types. Furthermore, we implemented two methods to generate valid upper and lower bounds for the green field design problem. For upper and lower bounding, we solved a restricted and a relaxed version of the problem, respectively. The first one, the restricted problem, is obtained by assuming a single type of passive splitter rather than multiple types. This reduces the number of variables in the model. Before starting the solution of the original problem, we solve this restricted problem for a maximum of $1800 \mathrm{~s}$ to obtain a good integer solution. This solution is fed into the original problem as a warm start. Therefore the original problem starts off the solution process by a good integer feasible solution. This effectively reduces the number of nodes to be visited in the branch and bound tree. For lower bounding, we solve the "Shortest Path Tree Problem" [53]. Observe that solving a shortest path tree rooted at central node provides a minimum distance network design without ensuring the QoS levels. Thus, it is a relaxation of the original problem and the bound it provides is sometimes an improvement over the Cplex bound.

\subsection{Key performance indicators and postprocessing}

For the key performance indicators (KPIs), we are primarily interested in the cost of setting up the network in both the problem types, which is a linear function of the fiber cable length. Furthermore, the quality of service (QoS) provided to the customers is another critical KPI. Thus, the average Internet speed provided to customers in terms of $\mathrm{MB} / \mathrm{s}$ ( $m$ variable) as well as $\mathrm{dB}$ values at each node ( $p$ variable) are important in the green field design problem. However, observe that the solution of the green field design problem might not provide the actual KPI values. Even though the constraints are satisfied, the KPI values we obtain from the problem might not be exact. For example, consider a leaf node $i$ that is directly connected to the central node. All the $m_{i}$ values in the range of [mbthreshold, mbcentral] satisfy the model constraints. However, the objective function of our model does not change for different service levels. Therefore, we need to perform a postprocessing on the network to obtain the actual KPI settings. In the postprocessing, we select the minimum port number if more than one splitter type satisfies the constraints. For the $m$ and $p$ values, we calculate the exact values using the system logic as shown in Constraints (8)-(11).

For the copper-field design problem, we guarantee that, with the utilization of fiber cables, the service levels for the consumers are better, the Internet is safer and the speed is faster than the copper-field case. On the other hand, the distance capacity parameter $H$ and the closeness proximity parameter $\gamma$ indicate the service quality for the consumers. These two parameters determine the combination of fiber and copper cables that consumers will be served with. Decreasing the $H$ value implies that the limit on the distance between the central node and the demand node decreases. Therefore the service level increases. With a similar reasoning, decreasing the $\gamma$ parameter increases the service level. With this in mind, we report the total fiber cable length, copper cable length in the network and the percentage of the nodes that are served by fiber cables as the quality of service indicators for the copper-field design problem.

\section{Computational results}

\subsection{Data}

To test the behavior of the mathematical models, extensive computational studies are performed. Rather than actual datasets, we were provided with two real-world representative datasets by the company due to commercial secrecy of the real data. Providing the solutions for these two realistic datasets was a certification of

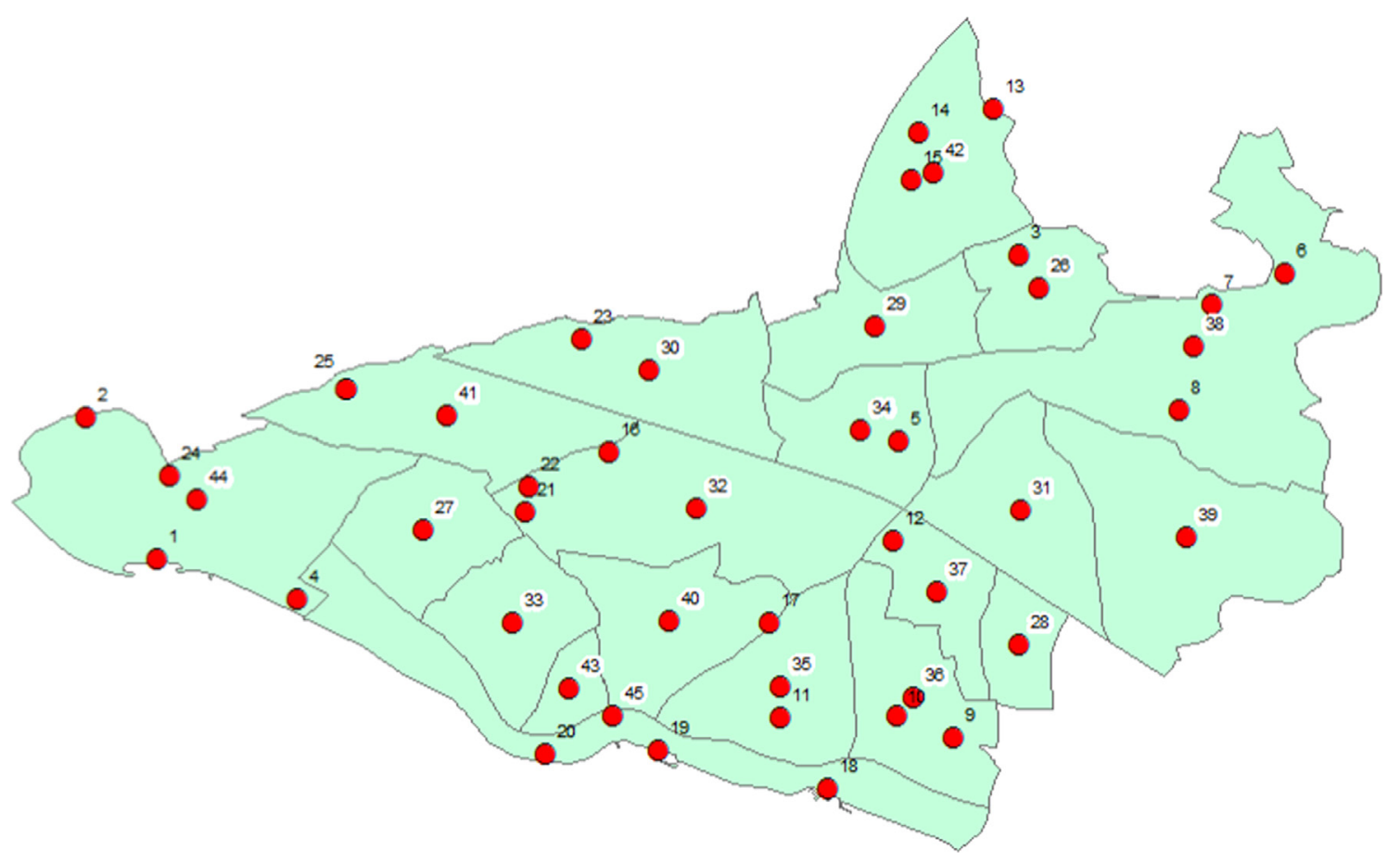

Fig. 3. Kartal map. 
our methodology which can be easily adapted to similar datasets by the company.

Two distinct datasets are of two districts in Istanbul. The small data set based on Kartal district involves 45 nodes, which includes private areas, business centers, shopping malls and public offices. The nodes represent the locations that need high bandwidth Internet access. Real shortest highway distances in kilometers attained from ArcGIS roadmap are utilized [51] for the $L$ matrix distance. The aggregated demand points, the central station (node 32 ) and the highways are depicted in Fig. 3. The maximum distance between any node pair is $11.23 \mathrm{~km}$. Other parameters related to Kartal dataset are shown in Table 1.

The larger data set based on Bakırköy district also includes private areas, business centers, shopping malls and public offices. The aggregated demand points can be seen in Fig. 4. The central station is node 74 . Distance parameters are shown in Table 2.

Apart from network data, the green field design problem requires several technical data which are provided to us by our service provider company (Table 3 ). In our particular application setting there are four passive splitter types with port numbers of $f_{1}=1, f_{2}=2, f_{3}=3$ and $f_{4}=4$. The costs associated with each passive splitter type are $s_{1}=\$ 12.5, s_{2}=\$ 15, s_{3}=\$ 20$ and $s_{4}=\$ 25$ and the cost of installing fiber cable is $\$ 1.00$ per meter. The speed of Internet at the central node is $2.5 \mathrm{~GB}$ and the threshold value is $100 \mathrm{MB} / \mathrm{s}$. The insertion loss in each level of port, declineps, is fixed at $3 \mathrm{~dB}$ for each port; the insertion loss per kilometer, declineway, is given as $0.2 \mathrm{~dB} / \mathrm{km}$ and the insertion loss budget, dBcapacity, is $28 \mathrm{~dB}$.

\subsection{Experimental design}

In an effort to provide the decision makers with alternatives, we experiment different designs for both green field and copper field design problems. For the green field problem, the $\mathrm{dB}$ values of the fiber cables, i.e. the dBCapacity, declineway and declineps, are

Table 1

Kartal dataset parameters.

\begin{tabular}{llll}
\hline Parameter & Min. & Max. & Avg. \\
\hline Distance between any two nodes $(\mathrm{km})$ & 0.21 & 11.23 & 4.79 \\
Distance from the central (node 32) $(\mathrm{km})$ & 1.22 & 7.38 & 4.02 \\
\hline
\end{tabular}

fixed for a given type of cable. Therefore, the only parameters that can be altered to change the key performance indicators (KPIs) are related to the Internet speed and the proportion of the costs of fiber cables and passive splitters. In this regard, we moderately alter the proportion between costs, namely, the alpha value. When the $\alpha$ value is 1 , it means that the model uses the given passive splitter costs and the fiber optical wiring costs. We also tested the $\alpha$ values of $0.00,0.25,0.50,0.75$ and 1.00 . Note that, as we have pointed out previously, the passive splitter cost is dominated by the cost of installing fiber links in today's technology [50]. Therefore, the realistic setting for the $\alpha$ value is 0.00 .

The baseline parameters for the out power of the central station, mbcentral, are $2.5 \mathrm{~GB}$ and the threshold Internet speed is $100 \mathrm{MB} / \mathrm{s}$. The service quality to the customers can change depending on the threshold speed. To see how the cost changes for different alternatives, we analyzed the cases for which the threshold value is $50 \mathrm{MB} / \mathrm{s}$ and $200 \mathrm{MB} / \mathrm{s}$.

Note that, all other parameters being equal, multiplying both the mbcentral and mbThreshold by the same constant does not change the optimal result of the green field design problem. For instance, the optimal network design of the case with

Table 2

Bakırköy dataset parameters.

\begin{tabular}{llll}
\hline Parameter & Min. & Max. & Avg. \\
\hline Distance between any two nodes $(\mathrm{km})$ & 0.14 & 11.98 & 5.02 \\
Distance from the central (node 74$)(\mathrm{km})$ & 1.67 & 8.96 & 6.41 \\
\hline
\end{tabular}

Table 3

Baseline parameter settings.

\begin{tabular}{ll}
\hline Parameter & Value \\
\hline central & 32 (Kartal), 74 (Bakırköy) \\
declineway & $0.2 \mathrm{~dB}$ \\
declineps & $3 \mathrm{~dB}$ \\
dBCapacity & $28 \mathrm{~dB}$ \\
mbcentral & $2.5 \mathrm{~GB} / \mathrm{s}$ \\
mbThreshold & $100 \mathrm{MB} / \mathrm{s}$ \\
\# passive splitter types & 4 \\
Passive splitter costs & $s_{1}=\$ 12.5, s_{2}=\$ 15, s_{3}=\$ 20, s_{4}=\$ 25$ \\
Fiber cable cost & $\$ 1 / \mathrm{m}$
\end{tabular}

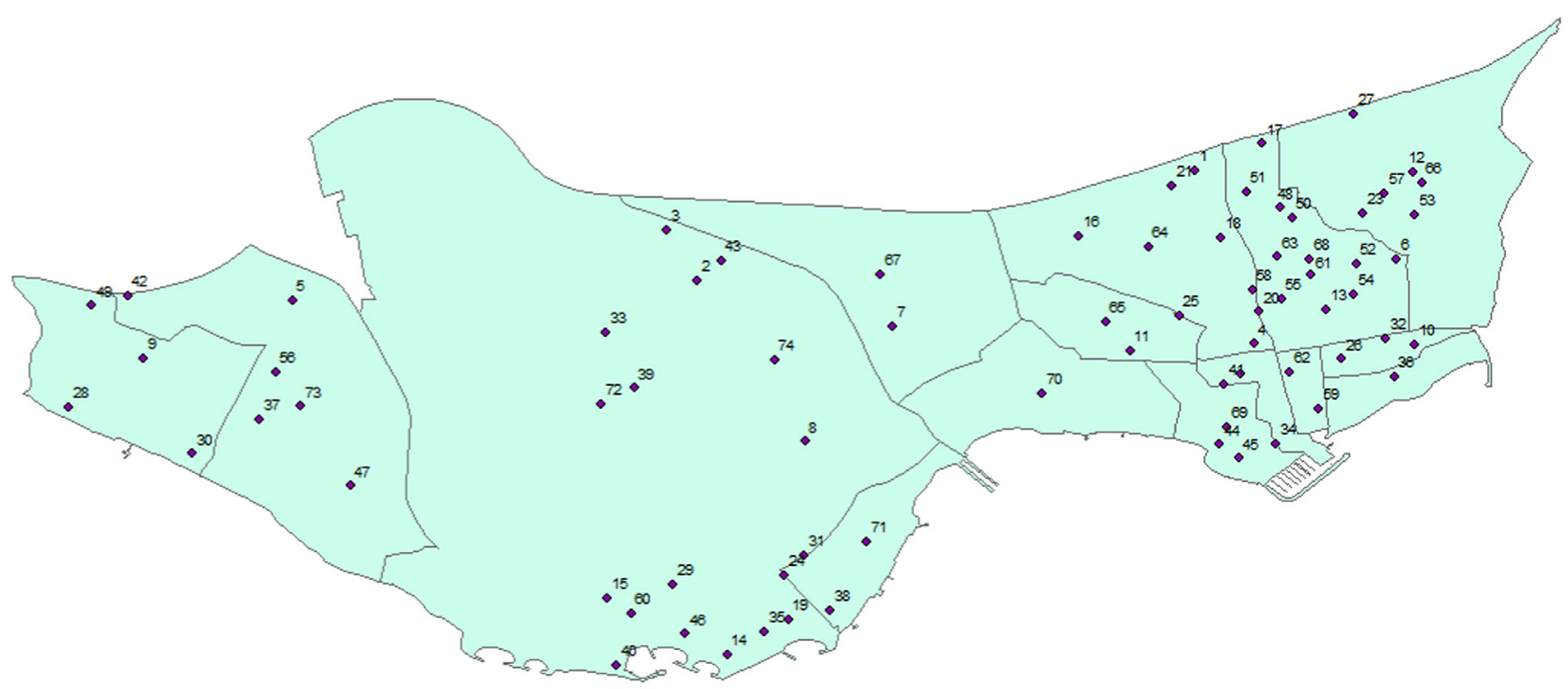

Fig. 4. Bakırköy map. 
Table 4

Equivalent cases for the experimental design.

\begin{tabular}{|c|c|c|c|c|c|c|c|c|}
\hline \multirow{2}{*}{$\begin{array}{l}\text { Instance } \\
\text { number }\end{array}$} & \multicolumn{2}{|c|}{ Experimental design } & \multicolumn{2}{|l|}{ Case1 } & \multicolumn{2}{|l|}{ Case2 } & \multicolumn{2}{|l|}{ Case3 } \\
\hline & mbcentral (GB) & mbThreshold (MB/s) & mbcentral (GB) & mbThreshold (MB/s) & mbcentral (GB) & mbThreshold (MB/s) & mbcentral (GB) & mbThreshold (MB/s) \\
\hline 1 & 2.5 & 50 & 1.25 & 25 & 5 & 100 & 10 & 200 \\
\hline 2 & 2.5 & 100 & 1.25 & 50 & 5 & 200 & 10 & 400 \\
\hline 3 & 2.5 & 200 & 1.25 & 100 & 5 & 400 & 10 & 800 \\
\hline
\end{tabular}

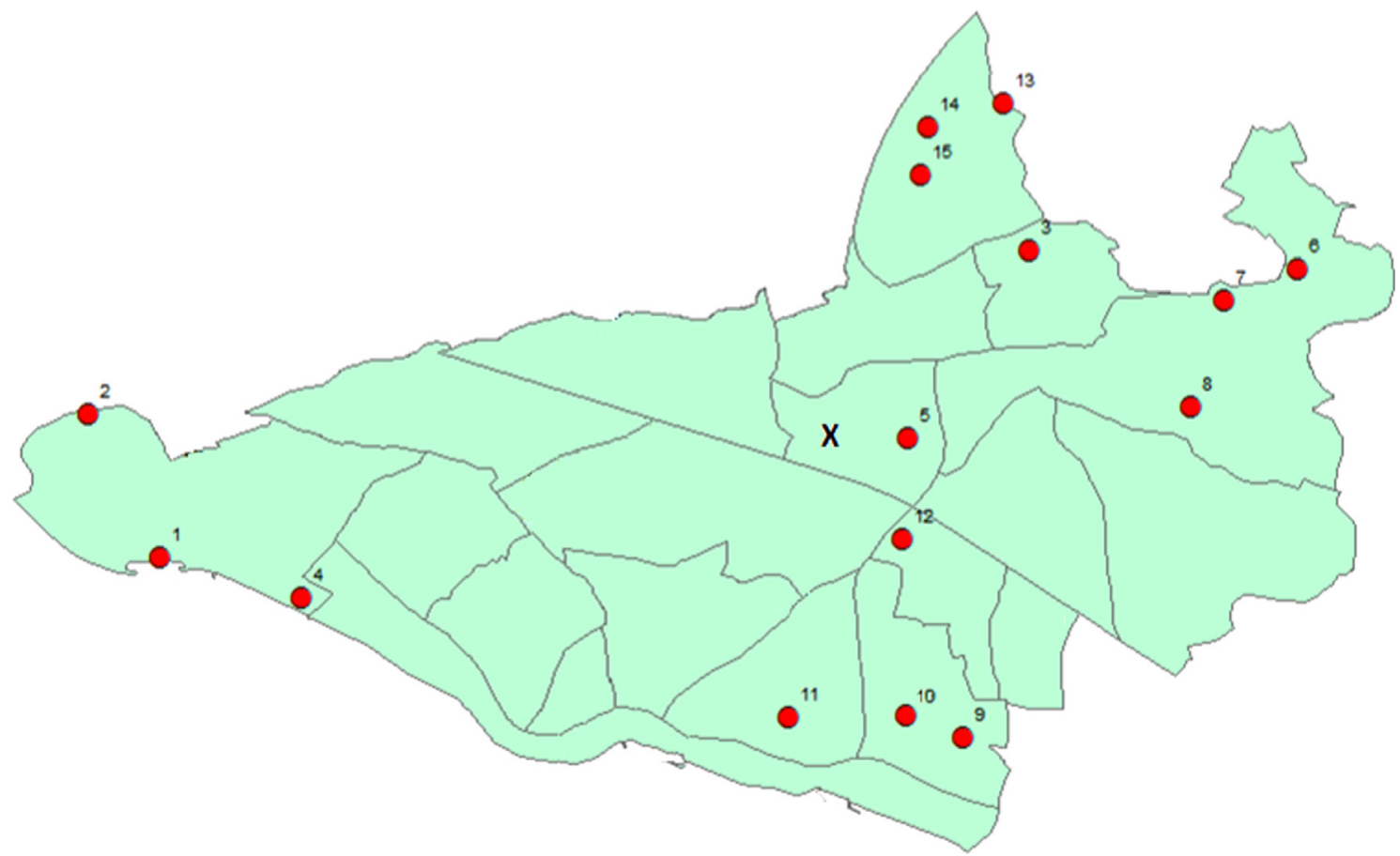

Fig. 5. Kartal network with 15 nodes and the geometric center marked.

mbcentral $=2.5 \mathrm{~GB}$ and $m b$ Threshold $=100 \mathrm{MB} / \mathrm{s}$ is also optimal for cases with mbcentral $=(2.5 k) \mathrm{GB}$ and mbThreshold $=(100 k) \mathrm{MB} / \mathrm{s}$, for all $k \geq 0$. Therefore, our experimental design provides answers to numerous other combinations, three of which are shown in Table 4.

For the copper field design problem, we consider different settings for the threshold distance $\gamma$ parameter, $H$ distance parameter and the number of cabinets $p$. In the experimental design, the $\gamma$ parameter changes in the $0-10 \mathrm{~km}$ range, the $p$ parameter changes between 2 and 8 , and the $H$ parameter changes between 10 and $50 \mathrm{~km}$.

Before presenting the above experimental design results, we consider different central node choices beside the actual central node to see how the location of the central node affects the results. For this reason, we constructed an artificial 15-node Kartal network and solved the green field design problem by choosing every node as the central node in different instances. In the following, we present the results of this analysis.

\subsection{The effect of the central node selection on the objective function}

In order to see how the central node selection affects the results, we constructed a sub-network of the Kartal dataset with 15 nodes (Fig. 5). The geometric center of the 15 nodes is marked with an ' $\mathrm{x}$ ' sign.

Table 5 shows the results of the green field design problem. Central node is the node selected as the central, and the ObjFnValue is the objective function value of the green field design problem with the selected central node and realistic choice of $\alpha=0$. Dist is the distance from the geometric center to the selected central node in kilometers. The Splitters column shows the types of passive splitters in use. The representation ' $a \times b$ ' shows that ath type passive splitter is used $b$ times in the network. The minimum, average and maximum MBs at the nodes are shown in the following three columns. Similarly, the minimum, average and maximum $\mathrm{dB}$ values are on the rightmost three columns.

Observe that the difference is not significant between different central node selections. The resulting designs change between 26.42 and $28.34 \mathrm{~km}$ in total. Since our cost is $\$ 1$ per meter, these numbers correspond to $\$ 26,420.9$ and $\$ 28,338.0$. As it can be observed in Fig. 5, the objective function value is related to how apart the selected node and the geometric center of the nodes are. For those nodes that are far apart from the geometric center (e.g. 1 and 2), the objective function values are the highest among all other nodes. Even though not perfectly linear, there is an obvious relationship between the distance from the center and the objective function value. Therefore, if there is any means of central node selection, geometric center of the nodes would produce 'good' results in terms of objective function value.

Fig. 6 plots the change in the objective function value (cost) versus the distance between the central and the geometric center. As we have noted above, being farther apart from the geometric center is an indication of higher costs. We can also spot this property in this figure. The three data points in top right part of the figure are those three nodes in the west part of the Kartal network in Fig. 5. 
Table 5

Green field design problem results of the 15-node network.

\begin{tabular}{|c|c|c|c|c|c|c|c|c|c|}
\hline Central node & $\begin{array}{l}\text { ObjFnValue } \\
\text { (thousand \$) }\end{array}$ & Dist (km) & Splitters & MinMB & AvgMB & MaxMB & MindB & AvgdB & MaxdB \\
\hline 1 & 28.12 & 7.49 & $1 \times 7$ & 156.25 & 791.67 & 2500 & 0.27 & 8.02 & 15.00 \\
\hline 2 & 28.34 & 7.12 & $1 \times 7$ & 156.25 & 791.67 & 2500 & 0.46 & 8.24 & 15.16 \\
\hline 3 & 26.90 & 3.20 & $1 \times 8$ & 156.25 & 989.58 & 2500 & 0.39 & 6.43 & 15.09 \\
\hline 4 & 27.70 & 7.14 & $1 \times 6$ & 156.25 & 875.00 & 2500 & 0.24 & 7.75 & 14.95 \\
\hline 5 & 26.60 & 1.86 & $1 \times 8$ & 156.25 & 854.17 & 2500 & 0.45 & 6.60 & 14.78 \\
\hline 6 & 27.79 & 4.56 & $1 \times 9$ & 156.25 & 770.83 & 2500 & 0.12 & 7.80 & 15.31 \\
\hline 7 & 27.33 & 4.15 & $1 \times 8$ & 156.25 & 1010.42 & 2500 & 0.12 & 6.71 & 15.21 \\
\hline 8 & 26.66 & 3.90 & $1 \times 9$ & 156.25 & 770.83 & 2500 & 0.38 & 7.77 & 15.08 \\
\hline 9 & 27.17 & 4.58 & $1 \times 8$ & 156.25 & 708.33 & 2500 & 0.15 & 8.18 & 14.23 \\
\hline 10 & 26.42 & 4.03 & $1 \times 7$ & 156.25 & 875.00 & 2500 & 0.10 & 7.28 & 14.13 \\
\hline 11 & 26.65 & 3.84 & $1 \times 7$ & 156.25 & 958.33 & 2500 & 0.28 & 6.87 & 14.19 \\
\hline 12 & 26.59 & 2.90 & $1 \times 8$ & 156.25 & 822.92 & 2500 & 0.44 & 6.83 & 13.75 \\
\hline 13 & 27.22 & 4.65 & $1 \times 7$ & 156.25 & 791.67 & 2500 & 0.21 & 7.52 & 15.14 \\
\hline 14 & 27.54 & 3.89 & $1 \times 5+2 \times 1$ & 156.25 & 875.00 & 2500 & 0.11 & 7.34 & 15.18 \\
\hline 15 & 27.02 & 3.34 & $1 \times 9$ & 156.25 & 697.92 & 2500 & 0.11 & 8.40 & 15.23 \\
\hline
\end{tabular}

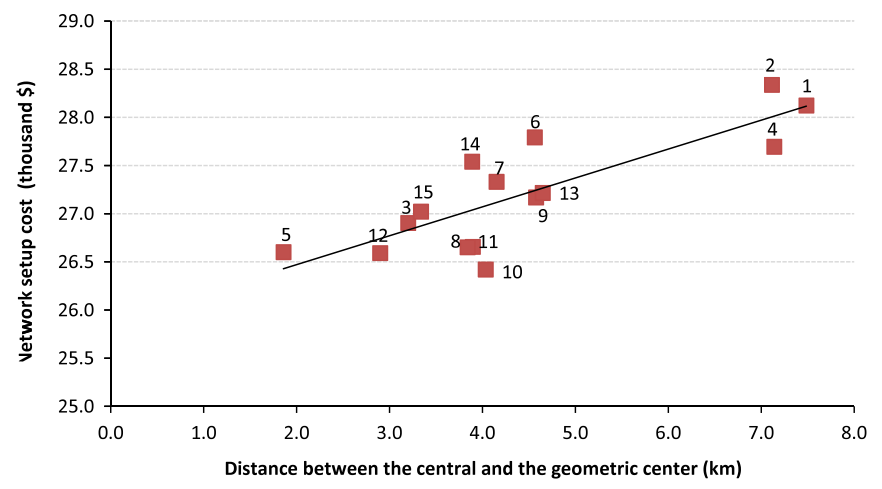

Fig. 6. Cost versus distance plot for different central nodes.

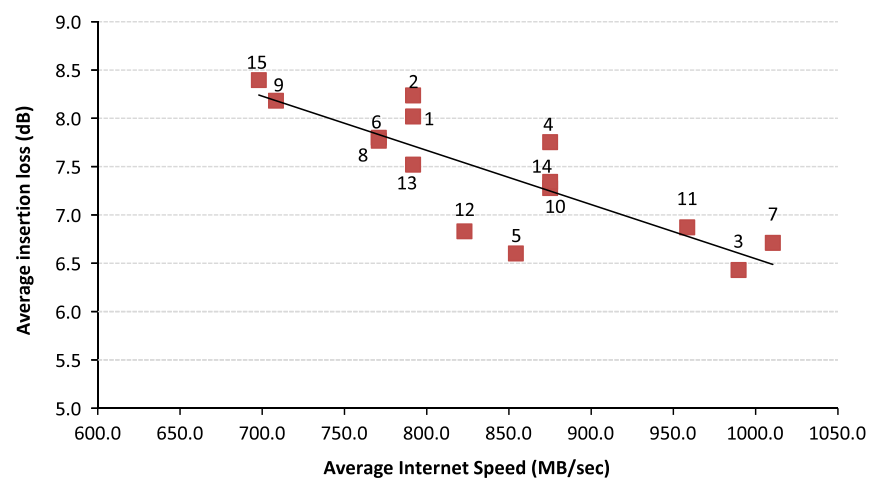

Fig. 7. Average Internet speed versus average insertion loss for different central nodes.

From the service perspective, the relationship between the average insertion loss and the average Internet speed is plotted in Fig. 7. Note that we can loosely claim that higher insertion loss implies lower Internet speed. Observe that average Internet speed decreases when there are more splitters on the route from the central to a given node. Encountering splitters also increases the insertion loss. Therefore, the service quality is proportional with the number of splitters in the network. Fewer number of splitters used is an indication of higher quality service.

\subsection{Green field model results}

Table 6 shows the green field model computational results. The parameter settings are shown in the 3 columns on the left side of the table. The mbcentral is $2.5 \mathrm{~GB}$ in each instance. The first column refers to the instance number, the second one is the $\alpha$ setting and the third column shows if the dataset is (K)artal or (B)akırköy. The last parameter column is the mbthreshold setting. Computational results are on the right side of the table. solTime refers to the total runtime of $10 \mathrm{~h}$ and the $0.5 \mathrm{~h}$ warmup period. A ' $\star$ ' symbol indicates 'out-of-memory' status of the model before the time limit. objFnVal refers to the best feasible solution obtained within the time limit. It is the total cost of fiber optic cable and passive splitters in U.S. dollars. Parentheses indicate that the solution is not optimal. lower bound is the lower bound obtained by Cplex except for those cases with a ' $\ddagger$ ' sign. In the instances with the sign, the lower bound provided by the shortest path tree (SPT) solution is better than the lower bound provided by Cplex. The last column shows the gap.

Even though 11 of the 15 Kartal instances were solved to optimality, the optimality gaps for the Bakırköy dataset change between $7.68 \%$ and $36.10 \%$. The lower bounds generated by the SPT solution improved Cplex lower bounds in Bakırköy instances for $\alpha$ value of zero. For the Kartal dataset, no improvement is obtained by the SPT bound.

Referring to Table 4, our experiments are equivalent to different settings. Note that improving from mbcentral $=2.5 \mathrm{~GB}$ and $m b$ Threshold $=50 \mathrm{MB} / \mathrm{s}$ setting to mbcentral $=5 \mathrm{~GB}$ and mbThreshold $=100 \mathrm{MB} / \mathrm{s}$ incurs no additional fiber cable cost since the network design for the former also satisfies the requirements of the latter design.

GK13, GK14 and GK15 network designs represent more realistic cases with an $\alpha$ parameter setting of zero in Kartal dataset. To emphasize the change in network designs for different threshold Internet speeds, we present these network designs as depicted in Fig. 8. Defining similarity of networks as the percentage of the arcs that are common in two given networks, Table 7 shows the similarity of the networks for Kartal dataset. Observe that GK13 and GK14 networks have more arcs in common than GK15 design.

Table 8 shows the splitter usage and the QoS results of the green field design problem for the GK13, GK14, GK15, GB13, GB14 and GB15 designs. The parameters are on the left side of the table. The Splitters column details the types of passive splitters used. The minimum, average and maximum MBs at the nodes are shown in the following three columns. Similarly, the minimum, average and maximum $\mathrm{dB}$ values are on the rightmost three columns. As expected, AvgMB increases for higher mbThreshold values.

Note that the QoS for customers in Kartal dataset is higher than that of Bakırköy dataset in terms insertion loss and Internet speed (Figs. 9 and 10). Since Kartal is a smaller district in size than Bakırköy, the route in Kartal network from the central to any other 
Table 6

Computational results for the green field design problem.

\begin{tabular}{|c|c|c|c|c|c|c|c|}
\hline \multicolumn{4}{|c|}{ Parameters } & \multicolumn{4}{|l|}{ Results } \\
\hline$\#$ & $\alpha$ & Dataset & mbthreshold & solTime & $\begin{array}{l}\text { objFnVal } \\
\text { (thousand\$) }\end{array}$ & Lower bound & $\begin{array}{l}\text { Gap } \\
(\%)\end{array}$ \\
\hline GK1 & 1.00 & K & 50 & 4.68 & 147.04 & 147.04 & 0.00 \\
\hline GK2 & 1.00 & $\mathrm{~K}$ & 100 & 4.68 & 147.04 & 147.04 & 0.00 \\
\hline GK3 & 1.00 & $\mathrm{~K}$ & 200 & 8.46 & 158.80 & 158.80 & 0.00 \\
\hline GK4 & 0.75 & K & 50 & 6.43 & 139.25 & 139.25 & 0.00 \\
\hline GK5 & 0.75 & $\mathrm{~K}$ & 100 & 6.33 & 139.25 & 139.25 & 0.00 \\
\hline GK6 & 0.75 & K & 200 & 12.29 & 147.38 & 147.38 & 0.00 \\
\hline GK7 & 0.50 & K & 50 & 18.21 & 125.11 & 125.11 & 0.00 \\
\hline GK8 & 0.50 & K & 100 & 25.30 & 125.11 & 125.11 & 0.00 \\
\hline GK9 & 0.50 & $\mathrm{~K}$ & 200 & 76.56 & 129.66 & 129.66 & 0.00 \\
\hline GK10 & 0.25 & $\mathrm{~K}$ & 50 & 236.22 & 99.99 & 99.99 & 0.00 \\
\hline GK11 & 0.25 & $\mathrm{~K}$ & 100 & 2154.61 & 99.99 & 99.99 & 0.00 \\
\hline GK12 & 0.25 & K & 200 & $36,986.32$ & $(105.30)$ & 102.66 & 2.51 \\
\hline GK13 & 0.00 & $\mathrm{~K}$ & 50 & $37,327.91$ & $(53.09)$ & 51.75 & 2.52 \\
\hline GK14 & 0.00 & K & 100 & $37,364.88$ & (54.57) & 51.69 & 5.27 \\
\hline GK15 & 0.00 & K & 200 & $37,837.61$ & (59.07) & 51.61 & 12.63 \\
\hline GB1 & 1.00 & B & 50 & $\star 10,597.76$ & $(233.60)$ & 215.65 & 7.68 \\
\hline GB2 & 1.00 & B & 100 & $\star 15,795.45$ & (233.26) & 214.99 & 7.83 \\
\hline GB3 & 1.00 & B & 200 & $\star 2208.72$ & $(271.24)$ & 249.80 & 7.90 \\
\hline GB4 & 0.75 & B & 50 & $\star 2076.65$ & (203.95) & 184.44 & 9.57 \\
\hline GB5 & 0.75 & B & 100 & $\star 1925.64$ & (204.96) & 183.46 & 10.49 \\
\hline GB6 & 0.75 & B & 200 & $\star 11,282.16$ & (236.24) & 212.37 & 10.10 \\
\hline GB7 & 0.50 & B & 50 & $\star 1503.25$ & $(171.96)$ & 152.84 & 11.12 \\
\hline GB8 & 0.50 & B & 100 & $\star 2277.58$ & (175.07) & 155.19 & 11.36 \\
\hline GB9 & 0.50 & B & 200 & $\star 3267.39$ & (199.32) & 169.47 & 14.98 \\
\hline GB10 & 0.25 & B & 50 & $\star 5696.37$ & (137.71) & 116.78 & 15.20 \\
\hline GB11 & 0.25 & B & 100 & $\star 5785.91$ & (142.67) & 117.77 & 17.45 \\
\hline GB12 & 0.25 & B & 200 & $\star 8729.91$ & $(166.71)$ & 119.83 & 28.12 \\
\hline GB13 & 0.00 & B & 50 & $37,823.85$ & $(68.81)$ & $\ddagger 53.48$ & 22.28 \\
\hline GB14 & 0.00 & B & 100 & $37,820.55$ & $(74.72)$ & $\mp 53.48$ & 28.42 \\
\hline GB15 & 0.00 & B & 200 & $37,823.87$ & $(83.70)$ & $\ddagger 53.48$ & 36.10 \\
\hline
\end{tabular}
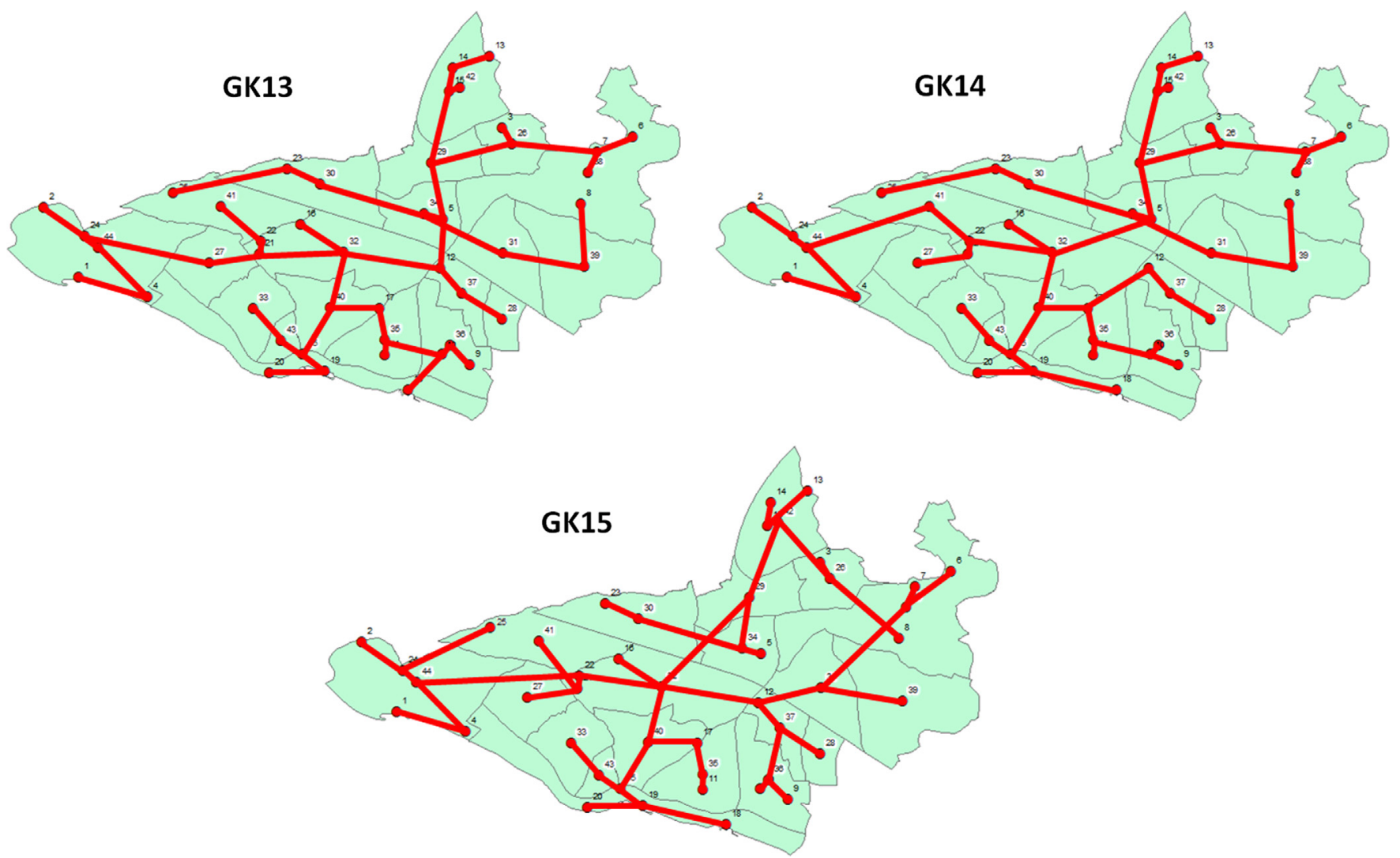

Fig. 8. Network designs for Kartal dataset. 
node includes fewer number of splitters than that in the Bakırköy network. Therefore the insertion loss is less and the Internet speed is higher in Kartal dataset.

Another observation is that, for a given network (Kartal or Bakırköy), the MaxdB decreases for increasing mbThreshold values (Fig. 9). This gives a clue about the network designs. For high values of mbThreshold, the network does not have many intermediate splitters on the route from the central to the leaf nodes to ensure that the threshold $\mathrm{MB}$ value requirement is met. However, when mbThreshold is low, then the route from the central to the leaf nodes may contain more splitters. Thus, the MaxdB is higher for those instances with low mbThreshold values. The Internet speed quality of each scenario is also depicted in Fig. 10.

Lastly, note that the green field network designs use type 1 or 2 passive splitters. However this is not always the case. Consider Instance GK2 (Fig. 11) in which one type 4 splitter is used in the network.

\subsection{Copper field re-design model results for Kartal}

In order to create a pre-built copper ring infrastructure, we use a nearest neighbor based greedy clustering algorithm. We fix a particular distance capacity as the maximum length of a copper ring. The algorithm starts with an arbitrary node and keeps visiting neighbors in a nearest neighbor fashion as long as the tour length does not exceed the specified distance capacity. When the threshold is exceeded, another ring infrastructure is constructed. Kartal region necessitated 4 copper rings with a distance capacity of $20 \mathrm{~km}$. Note that none of the copper rings include the central station.

We increase the $\gamma$ value starting from zero with equal intervals up to the point where with the $\gamma$ coverage, all demand nodes are served without any additional fiber links. This value is $10 \mathrm{~km}$ for the Kartal data set. There is no need to increase the $\gamma$ value beyond this value since the objective function value will not change. Fig. 12 depicts the effect of the $\gamma$ value over the network design for the Kartal data. The solution of the copper field re-design problem also depends on the number of cabinets (parameter $p$ ) that need to be installed. When we add 8 cabinets into the Kartal data, all nodes can be covered without any cabinet-node links (for $\gamma=5 \mathrm{~km}$ ). For the analysis, we used the midpoint $\gamma$ value of $5 \mathrm{~km}$ of the previous analysis. The distance threshold value is taken as $50 \mathrm{~km}$, which corresponds to practically the uncapacitated case.

Table 7

Similarity of network designs.

\begin{tabular}{llll}
\hline $\begin{array}{l}\text { Network } \\
\text { design }\end{array}$ & GK13 (\%) & GK14 (\%) & GK15 (\%) \\
\hline GK13 & 100.0 & 75.0 & 50.0 \\
GK14 & 75.0 & 100.0 & 54.6 \\
GK15 & 50.0 & 54.6 & 100.0 \\
\hline
\end{tabular}

Fig. 13 shows the decrement in the number of cabinet-node links, as the number of cabinets increases. When there are 8 cabinets, there is no need for any fiber link in this data set.

The results are also sensitive to the distance threshold value in kilometers (parameter $H$ ). In this analysis, we again use $\gamma$ value as $5 \mathrm{~km}$. Distance threshold values are taken as 10,20 and $30 \mathrm{~km}$ for the Kartal region. Beyond this value, there is no change in the objective function value. As the threshold decreases, the infrastructure of the network changes. The results depicted in Table 9 are for $\gamma=5 \mathrm{~km}, p=2,4,6,8$ and $H=10,20,30,40,50 \mathrm{~km}$. The last three columns in Table 9 depict the fiber and copper cable lengths and the percentage and the number of nodes that are served by fiber cables. Note that the length of the fiber cable results in a setup cost for the company, whereas the copper cable and the percentage of the nodes served by the fiber cables are the QoS parameters for the consumers. Since the consumers would rather prefer to be served by fiber rather than copper cables due to increased safety and speed, higher values for the fiber cable length and for the nodes that are served by the fiber cables indicate higher QoS levels. Note that we guarantee a better service level for the consumers by using fiber cables at least partially in the network. However increasing cable lengths implies much safer Internet and the speed is faster than the copper-field case.

For the analysis of different $\gamma$ values, we ranged $\gamma$ as $1,3,5$, 7 and $10 \mathrm{~km}$. Tables 9 and 10 depict the results. We omit the instances after break points of the corresponding distance threshold values beyond which the objective value remains the same; i.e., $H$ after $20 \mathrm{~km}$ for $p=2,4$ and $H$ after $30 \mathrm{~km}$ for $p=6,8$ where $\gamma=1,3 \mathrm{~km}$; similarly, $H$ after $20 \mathrm{~km}$ remains the same when $\gamma$ is 7 and $10 \mathrm{~km}$.

Observe from Table 10 that, when we increase $\gamma$, for fixed $p$ and $H$, the objective function value, which is directly related with fiber link, decreases. For example when we compare Instances CK 21 and $\mathrm{CK} 33$, the cabinets can cover more demand nodes due to the increase in the cover threshold. Since the coverage increases, the cabinet-node links decrease. Hence, the objective function value decreases. Similarly, the objective function value decreases from

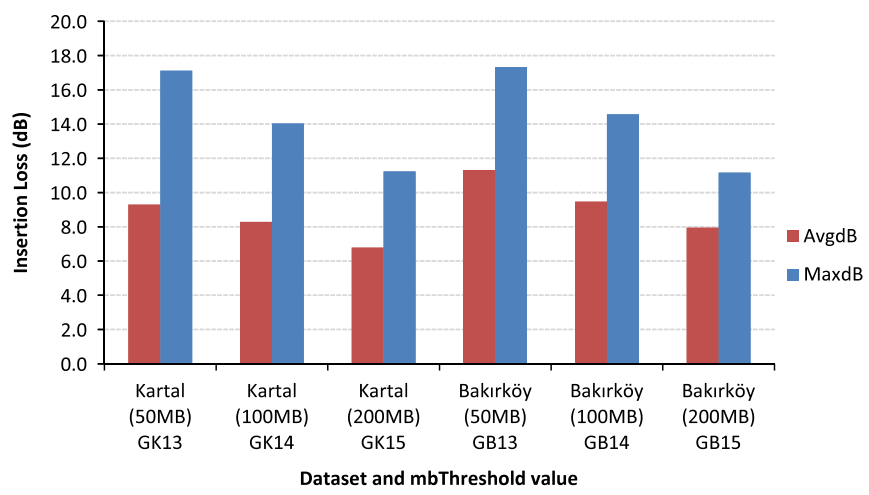

Fig. 9. Insertion loss for different scenarios.

Table 8

Quality of service results for the green field design problem.

\begin{tabular}{|c|c|c|c|c|c|c|c|c|c|}
\hline \multicolumn{3}{|c|}{ Parameters } & \multicolumn{7}{|l|}{ Results } \\
\hline$\#$ & Dataset & mbThreshold & Splitters & MinMB & AvgMB & MaxMB & MindB & AvgdB & MaxdB \\
\hline GK13 & K & 50 & $1 \times 27$ & 78.13 & 626.78 & 2500 & 0.25 & 9.50 & 17.12 \\
\hline GK14 & K & 100 & $1 \times 26$ & 156.25 & 674.72 & 2500 & 0.24 & 8.47 & 14.03 \\
\hline GK15 & K & 200 & $1 \times 22$ & 312.50 & 838.07 & 2500 & 0.24 & 6.94 & 11.23 \\
\hline GB13 & B & 50 & $2 \times 1+1 \times 41$ & 78.13 & 441.99 & 2500 & 0.33 & 11.47 & 17.32 \\
\hline GB14 & B & 100 & $2 \times 1+1 \times 38$ & 156.25 & 586.47 & 2500 & 0.33 & 9.60 & 14.57 \\
\hline GB15 & $\mathrm{B}$ & 200 & $1 \times 35$ & 312.50 & 723.46 & 2500 & 0.33 & 8.05 & 11.16 \\
\hline
\end{tabular}


Instance CK 25 to $\mathrm{CK}$ 37. On the consumers side, the percentage of the nodes that are served by fiber cables decreases for higher $\gamma$ and $p$ values, since these parameters indicate the number of nodes that can be covered by a given cabinet using the fiber cables.

The effect of the number of cabinets can be observed at Instances CK 3, CK 8, CK 13 and CK 18 of Table 9. For fixed $\gamma=5 \mathrm{~km}$ and $H=30 \mathrm{~km}$, the increase in the number of cabinets provides more coverage from the cabinets. Hence, the objective function value decreases.

Another observation is about the sensitivity of the objective function value to the number of cabinets. When the $\gamma$ value is $10 \mathrm{~km}$ and distance threshold value is $10 \mathrm{~km}$, the objective function value changes for different numbers of cabinets. When the number of cabinets is 2, the objective function value is $76.91 \mathrm{~km}$, in Instance CK 53 as shown in Fig. 14. Observe here that even though there are many other nodes which are still close enough (within $\gamma$ ) to the cabinets, they still need a fiber access since they do not have a copper link to the opened cabinets. However, 4 cabinets can cover all nodes without the need for any fiber cabinet-node link.

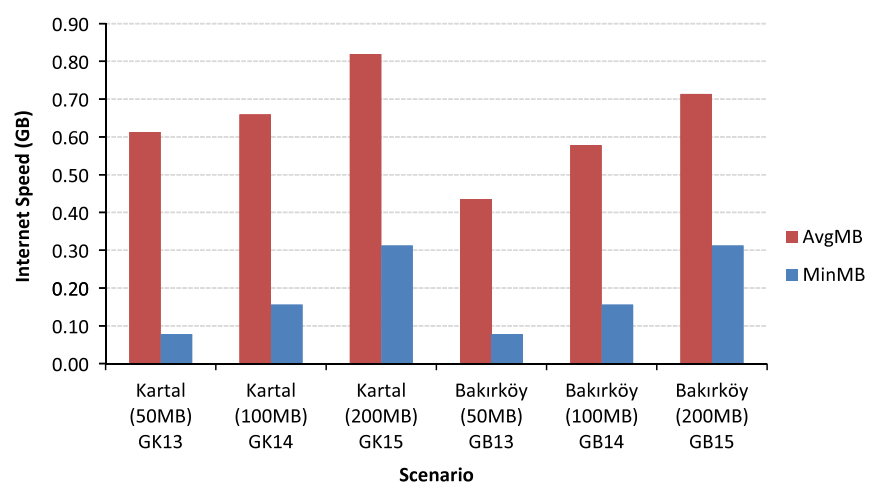

Fig. 10. Internet speed for different scenarios.
Interestingly, when we continue to increase the number of cabinets, the objective function starts to increase. From Instance CK 55 to Instance CK 57, the number of cabinets increases by 2 . In Instance $\mathrm{CK}$ 57, there are 6 cabinets and the ring around cabinets needs more usage of fiber optical wire which causes additional cost in the objective function value.

Finally, when we add 2 more cabinets to Instance CK 59, the objective function value rises to $19.94 \mathrm{~km}$. This increase in the objective function value shows that the network does not need that many cabinets.

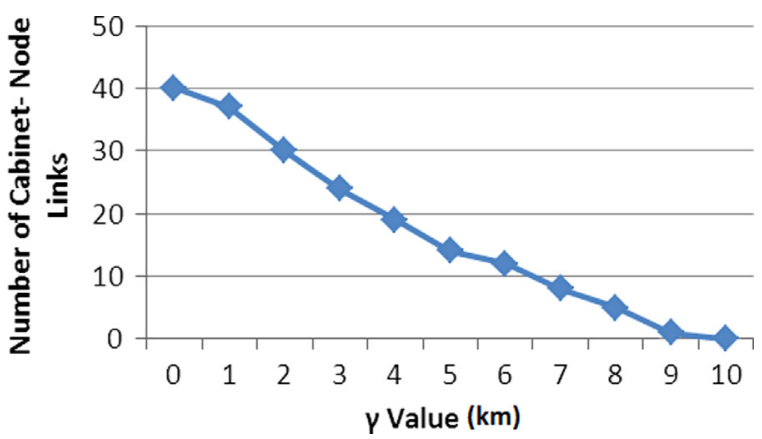

Fig. 12. Effect of $\gamma$ value.

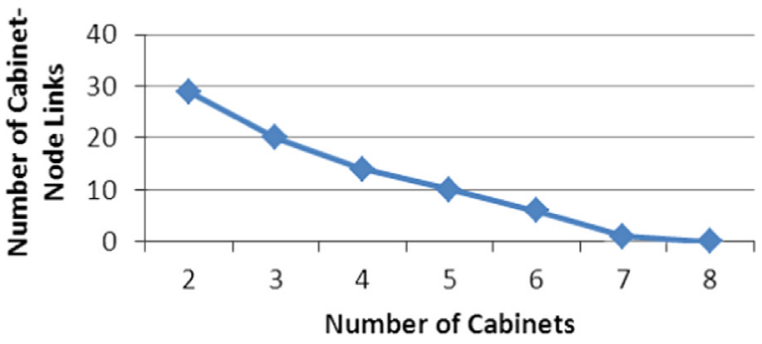

Fig. 13. Effect of number of cabinets.

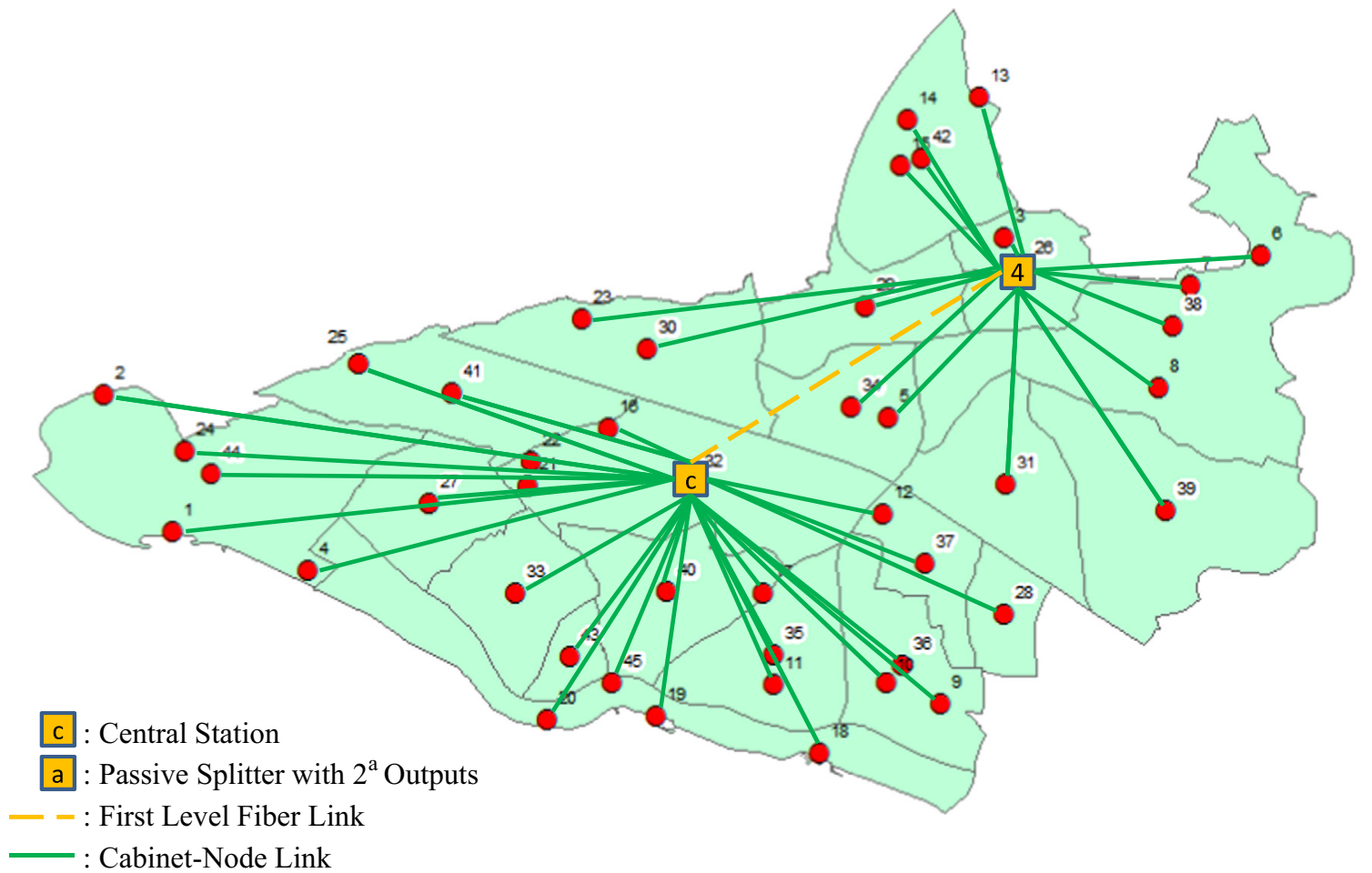

Fig. 11. Network design for Kartal dataset with $\alpha=1$. 
Table 9

Effect of distance threshold.

\begin{tabular}{|c|c|c|c|c|c|c|c|}
\hline \multirow[t]{2}{*}{$\#$} & \multicolumn{3}{|c|}{ Parameters } & \multicolumn{4}{|l|}{ Results } \\
\hline & $\begin{array}{l}\gamma \\
(\mathrm{km})\end{array}$ & $p$ & $\begin{array}{l}H \\
(\mathrm{~km})\end{array}$ & $\mathrm{CPU}(\mathrm{s})$ & $\begin{array}{l}\text { Fiber cable } \\
\text { length (Obj. } \\
\text { Fn.Val.) } \\
(\mathrm{km})\end{array}$ & $\begin{array}{l}\text { Copper } \\
\text { cable } \\
\text { length } \\
(\mathrm{km})\end{array}$ & $\begin{array}{l}\text { Percentage and } \\
(\#) \text { of nodes } \\
\text { served by fiber } \\
\text { cables }\end{array}$ \\
\hline CK1 & 5 & 2 & 10 & 0.07 & 115.19 & 33.23 & $71.11 \%(32)$ \\
\hline CK2 & & & 20 & 9.25 & 93.72 & 37.66 & $71.11 \%(32)$ \\
\hline CK3 & & & 30 & 11.31 & 93.72 & 37.66 & $71.11 \%(32)$ \\
\hline CK4 & & & 40 & 9.77 & 93.72 & 37.66 & $71.11 \%(32)$ \\
\hline CK5 & & & 50 & 10.51 & 93.72 & 37.66 & $71.11 \%(32)$ \\
\hline CK6 & & 4 & 10 & 92.6 & 60.62 & 68.50 & $46.67 \%(21)$ \\
\hline CK7 & & & 20 & 11.76 & 51.78 & 76.40 & $42.22 \%(19)$ \\
\hline CK8 & & & 30 & 9.64 & 51.78 & 76.40 & $42.22 \%(19)$ \\
\hline СК9 & & & 40 & 10.93 & 51.78 & 76.40 & $42.22 \%(19)$ \\
\hline CK10 & & & 50 & 9.19 & 51.78 & 76.40 & $42.22 \%(19)$ \\
\hline CK11 & & 6 & 10 & 719.26 & 41.52 & 89.39 & $31.11 \%$ (14) \\
\hline CK12 & & & 20 & 88.19 & 35.51 & 99.93 & $24.44 \%(11)$ \\
\hline CK13 & & & 30 & 19.23 & 33.79 & 94.11 & $26.67 \%(12)$ \\
\hline CK14 & & & 40 & 66.17 & 33.79 & 94.11 & $26.67 \%(12)$ \\
\hline CK15 & & & 50 & 50.94 & 33.79 & 94.11 & $26.67 \%(12)$ \\
\hline CK16 & & 8 & 10 & 9353.21 & 36.78 & 106.08 & $22.22 \%(10)$ \\
\hline CK17 & & & 20 & 305.08 & 28.59 & 105.91 & $20.00 \%(9)$ \\
\hline CK18 & & & 30 & 53.52 & 25.30 & 106.89 & $20.00 \%(9)$ \\
\hline CK19 & & & 40 & 57.11 & 25.30 & 106.89 & $20.00 \%(9)$ \\
\hline CK20 & & & 50 & 55.57 & 25.30 & 106.89 & $20.00 \%(9)$ \\
\hline
\end{tabular}

From the consumers' perspective, the copper cable length increases for increasing $\gamma$ and $p$ values. When the $\gamma$ parameter equals 1 and the number of cabinets is 2 , then all of the nodes are served by fiber cables. On the other end, when the $\gamma$ parameter equals 10 and the number of cabinets is 4 , the percentage of the nodes that are served by fiber cables hits a bottom value of $11.11 \%$. Even though increasing the $\gamma$ parameter might be considered as a good opportunity for the company to decrease the costs, it is not a good practice for the consumers since the number of consumers that are served by the fiber cables decreases.

Up until now, we analyzed the performance of the models when there are no premise nodes in the data set (i.e., $P=\varnothing$ ). We now provide some results including premise nodes. For these analyses, we select three clusters of premise nodes: the first one is 7,38 , the second one is $7,9,10,36,38$ and the last one is $1,2,7,9$, $10,24,36,38,44$. For the computational analyses, we use three different values for each of the parameters $\gamma, p$ and $H$. Our findings are summarized in Table 11.

The first observation is about the objective function value. As the number of premise nodes increases, the number of fiber links between premise nodes increases. For example, Instance CKP 4 has 2 premise nodes. The objective value is $34.68 \mathrm{~km}$. When we add 3 more premise nodes (Instance CKP 5), the objective function value rises to $40.79 \mathrm{~km}$. For the final one, where premise nodes are $1,2,7,9,10,24,36,38,44$ in Instance CKP 6 , the objective function value increases to $48.69 \mathrm{~km}$. Similar observations can be made about increases in the $\gamma$ value and in the number of cabinets in these instances with premise nodes. From Instance CKP 2 to Instance CKP 5, both the $\gamma$ value and $p$ increase whereas premise nodes remain the same. Despite the presence of premise nodes, as the $\gamma$ value and the number of cabinets increase from 0 to 5 and 4 to 6 , respectively, more demand nodes can be covered. Hence, the need for cabinet-node links, and ultimately, the objective function value decreases.

For comparison purposes, we refer to Fig. 15, where $\gamma$ is $10 \mathrm{~km}$, $p$ is 8 and $H$ is $10 \mathrm{~km}$. When we add two premise nodes 7 and 38, the objective function value increases from $19.94 \mathrm{~km}$ (Instance CK
Table 10

Copper field results for Kartal dataset.

\begin{tabular}{|c|c|c|c|c|c|c|c|}
\hline \multirow[t]{2}{*}{ \# } & \multicolumn{3}{|c|}{ Parameters } & \multicolumn{4}{|l|}{ Results } \\
\hline & $\begin{array}{l}\gamma \\
(\mathrm{km})\end{array}$ & $p$ & $\begin{array}{l}H \\
(\mathrm{~km})\end{array}$ & $\mathrm{CPU}(\mathrm{s})$ & $\begin{array}{l}\text { Fiber cable } \\
\text { length (Obj. } \\
\text { Fn.Val.) } \\
(\mathrm{km})\end{array}$ & $\begin{array}{l}\text { Copper } \\
\text { cable } \\
\text { length } \\
(\mathrm{km})\end{array}$ & $\begin{array}{l}\text { Percentage and } \\
(\#) \text { of nodes } \\
\text { served by fiber } \\
\text { cables }\end{array}$ \\
\hline CK21 & 1 & 2 & 10 & 0.15 & 136.79 & 0.00 & $100.00 \%(45)$ \\
\hline CK22 & & & 20 & 6.21 & 119.49 & 0.00 & $100.00 \%(45)$ \\
\hline CK23 & & & 30 & 5.54 & 119.49 & 0.00 & $100.00 \%(45$ \\
\hline CK24 & & 4 & 10 & 70.65 & 96.98 & 1.85 & $95.56 \%(43)$ \\
\hline CK25 & & & 20 & 9.79 & 89.39 & 3.08 & $91.11 \%(41)$ \\
\hline CK26 & & & 30 & 8.86 & 89.39 & 3.08 & $91.11 \%(41)$ \\
\hline CK27 & & 6 & 10 & 5519.15 & 87.49 & 5.27 & $84.44 \%(38)$ \\
\hline CK28 & & & 20 & 131.73 & 78.43 & 6.04 & $80.00 \%(36)$ \\
\hline CK29 & & & 30 & 14.24 & 76.14 & 7.46 & $75.56 \%(34)$ \\
\hline СК30 & & 8 & 10 & 4183.16 & 81.28 & 8.11 & $75.56 \%(34)$ \\
\hline CK31 & & & 20 & 581.13 & 69.99 & 9.03 & $71.11 \%(32)$ \\
\hline CK32 & & & 30 & 265.41 & 67.54 & 10.27 & $66.67 \%(30)$ \\
\hline CK33 & 3 & 2 & 10 & 0.05 & 125.85 & 13.87 & $84.44 \%(38)$ \\
\hline CK34 & & & 20 & 6.26 & 107.17 & 18.45 & $80.00 \%(36)$ \\
\hline CK35 & & & 30 & 7.29 & 107.17 & 18.45 & $80.00 \%(36)$ \\
\hline CK36 & & 4 & 10 & 14.85 & 79.61 & 24.83 & $66.67 \%(30)$ \\
\hline CK37 & & & 20 & 10.91 & 72.26 & 27.38 & $64.44 \%(29)$ \\
\hline CK38 & & & 30 & 9.15 & 72.26 & 27.38 & $64.44 \%(29)$ \\
\hline CK39 & & 6 & 10 & 636.77 & 62.55 & 45.06 & $44.44 \%(20)$ \\
\hline CK40 & & & 20 & 61.74 & 55.5 & 45.06 & $44.44 \%(20)$ \\
\hline CK41 & & & 30 & 19.27 & 52.77 & 45.06 & $44.44 \%(20)$ \\
\hline CK42 & & 8 & 10 & 5081.97 & 55.05 & 48.94 & $40.00 \%(18)$ \\
\hline CK43 & & & 20 & 62.36 & 42.01 & 51.51 & $33.33 \%(15)$ \\
\hline CK44 & & & 30 & 9.70 & 39.28 & 51.51 & $33.33 \%(15)$ \\
\hline CK45 & 7 & 2 & 10 & 0.07 & 109.57 & 57.43 & $64.44 \%(29)$ \\
\hline CK46 & & & 20 & 9.71 & 80.22 & 80.83 & $55.56 \%(25)$ \\
\hline CK47 & & 4 & 10 & 12.37 & 39.46 & 137.51 & $28.89 \%(13)$ \\
\hline CK48 & & & 20 & 11.97 & 35.40 & 129.44 & $28.89 \%(13)$ \\
\hline CK49 & & 6 & 10 & 330.67 & 27.19 & 124.19 & $20.00 \%(9)$ \\
\hline CK50 & & & 20 & 96.57 & 22.89 & 143.32 & $20.00 \%(9)$ \\
\hline CK51 & & 8 & 10 & $10,878.32$ & 27.20 & 123.06 & $20.00 \%(9)$ \\
\hline CK52 & & & 20 & 168.34 & 19.72 & 133.37 & $20.00 \%(9)$ \\
\hline CK53 & 10 & 2 & 10 & 0.03 & 76.91 & 137.45 & $46.67 \%(21)$ \\
\hline CK54 & & & 20 & 4.72 & 55.50 & 139.77 & $42.22 \%(19)$ \\
\hline CK55 & & 4 & 10 & 5.01 & 17.75 & 211.80 & $11.11 \%(5)$ \\
\hline CK56 & & & 20 & 3.40 & 14.40 & 213.44 & $11.11 \%(5)$ \\
\hline CK57 & & 6 & 10 & 61.37 & 17.81 & 185.39 & $15.56 \%(7)$ \\
\hline CK58 & & & 20 & 27.45 & 14.99 & 169.70 & $15.56 \%(7)$ \\
\hline CK59 & & 8 & 10 & 455.12 & 19.94 & 195.11 & $20.00 \%(2)$ \\
\hline CK60 & & & 20 & 199.06 & 16.05 & 156.40 & $20.00 \%(9)$ \\
\hline
\end{tabular}

Table 11

Copper field with premise nodes for Kartal dataset.

\begin{tabular}{|c|c|c|c|c|c|c|}
\hline \multirow[t]{2}{*}{$\#$} & \multicolumn{4}{|c|}{ Parameters } & \multicolumn{2}{|l|}{ Results } \\
\hline & $\gamma$ & $p$ & $H$ & Premise nodes & $\begin{array}{l}\text { Objective function } \\
\text { value }\end{array}$ & $\begin{array}{l}\text { CPU } \\
(\mathrm{s})\end{array}$ \\
\hline CKP1 & 0 & 4 & 50 & 7,38 & 92.39 & 7.91 \\
\hline CKP2 & & & & $7,9,10,36,38$ & 93.12 & 6.78 \\
\hline CKP3 & & & & $1,2,7,9,10,24,36,38,44$ & 95.56 & 7.24 \\
\hline CKP4 & 5 & 6 & 30 & 7,38 & 34.68 & 14.11 \\
\hline CKP5 & & & & $7,9,10,36,38$ & 40.79 & 16.84 \\
\hline CKP6 & & & & $1,2,7,9,10,24,36,38,44$ & 48.49 & 14.05 \\
\hline CKP7 & 10 & 8 & 10 & 7,38 & 22.71 & 1500.92 \\
\hline CKP8 & & & & $7,9,10,36,38$ & 29.14 & 4644.87 \\
\hline СКР9 & & & & $1,2,7,9,10,24,36,38,44$ & 41.37 & 6173.74 \\
\hline
\end{tabular}

59) to $22.71 \mathrm{~km}$ (Instance CKP 7). This increase is due to the fiber links between premise nodes as shown in Fig. 16.

Interestingly, in addition to nodes 7 and 38, when we add nodes $9,10,36$ to the premise node set, the cabinet locations change in order to decrease the total fiber length (Fig. 17). 


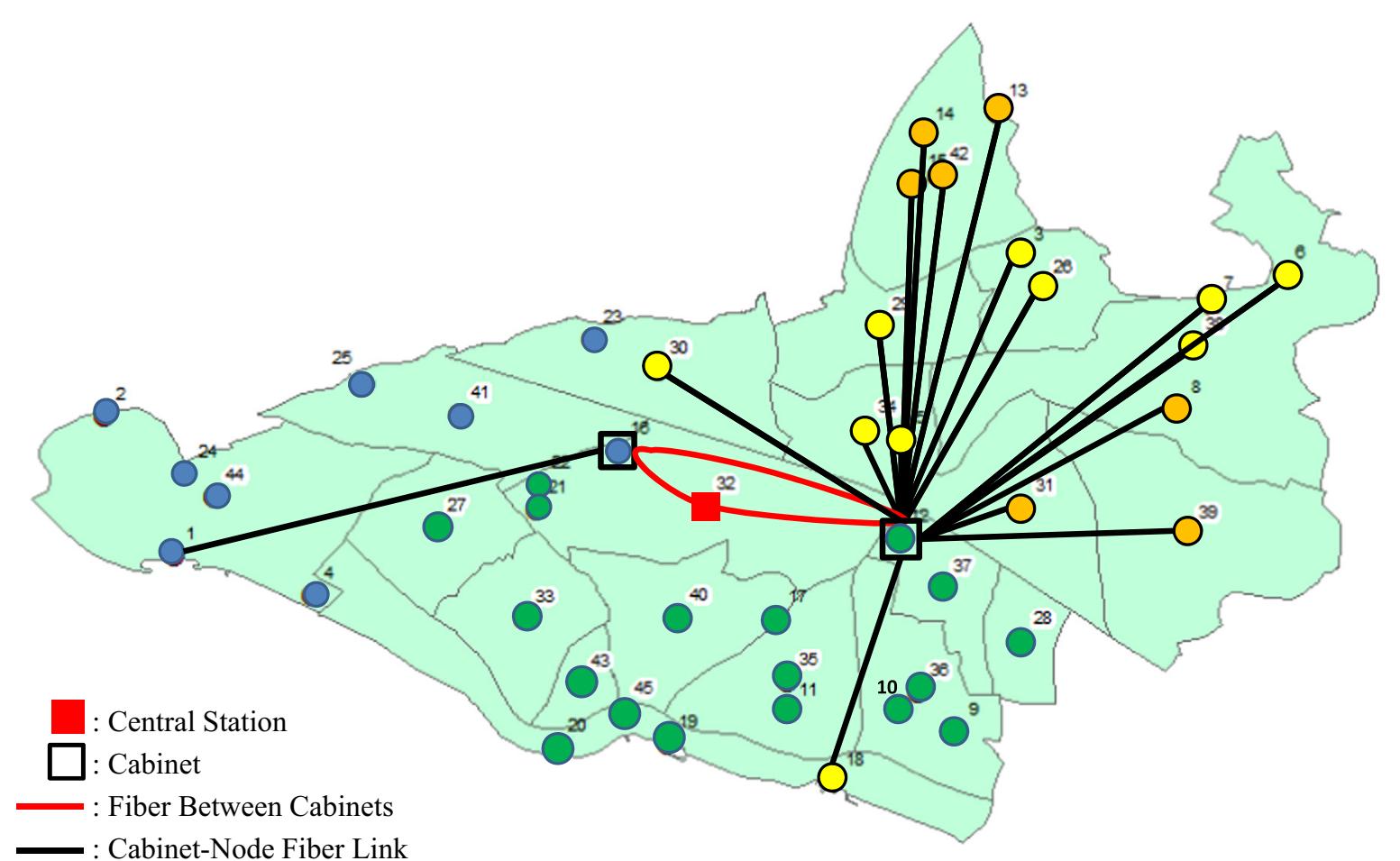

Fig. 14. Illustration of instance CK 53.

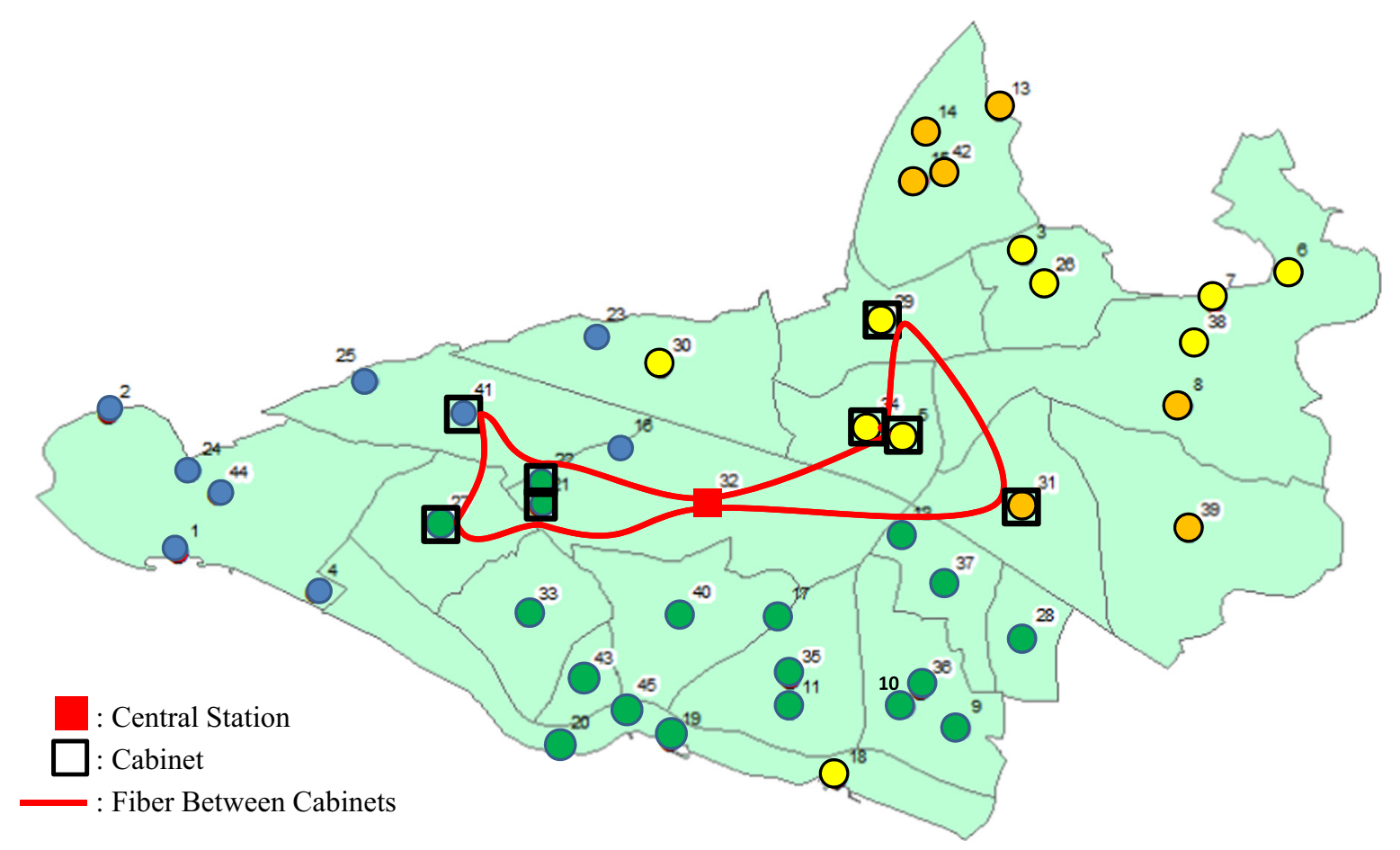

Fig. 15. Illustration of instance CK 59.

From Instance CKP 8 to CKP 9, we only change the premise node set by adding nodes $1,2,24$, and 44 . For this instance, two of the cabinet locations change. Also, new fiber links between the premise nodes are added.

\subsection{Copper field model results for Bakırköy data set}

For the construction of copper rings, we adopted the same approach used for Kartal data set. Since the Bakırköy data set is dense, its maximum length is smaller than that in the Kartal data set and the maximum length of a copper ring is $15 \mathrm{~km}$. The algorithm results in 6 copper rings.

For this data set, we varied $p$ (number of cabinets) as 3 and 6 . The $\gamma$ value is selected from $\{0,1,5,10\} \mathrm{km}$. The $H$ values are larger when compared to Kartal data. Also, due to the structure of the demand node locations, the length of the ring between cabinets needs to be longer. The results are summarized in Table 12. There are three instances which could not be solved to optimality after 


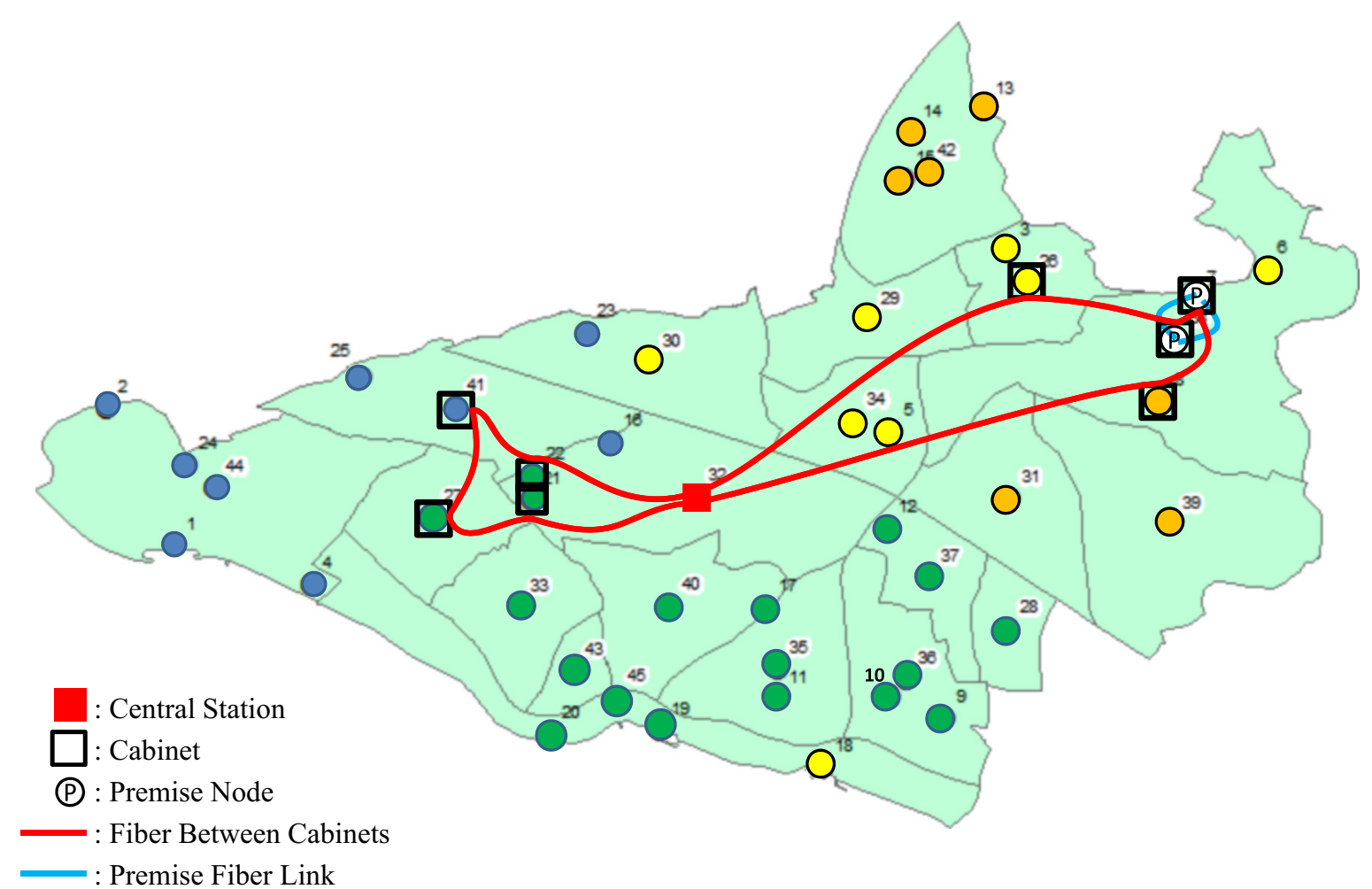

Fig. 16. Illustration of instance CKP 7.

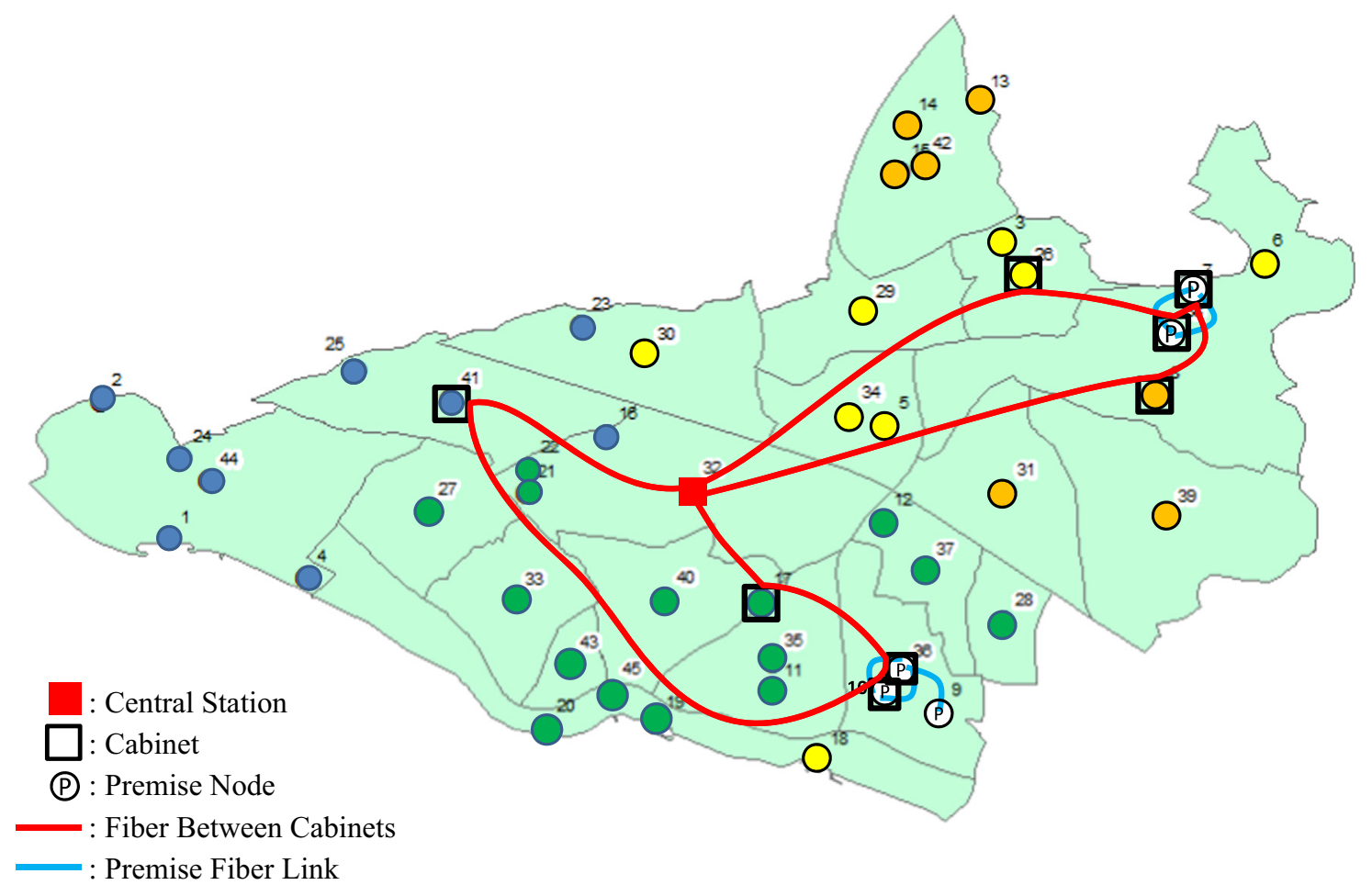

Fig. 17. Illustration of instance CKP 8.

$0.5 \mathrm{~h}$ warm-up period and $10 \mathrm{~h}$ of runtime. The gaps when Cplex terminated are actually higher than what is reported in the table. But we have a better lower bound also provided in the table. The result we obtained in CB6 instance is a lower bound for the CB5 instance, since the only difference is the $H$ parameter. We know that decreasing $H$ value from 20 to 15 cannot improve the objective function value from 103.88. Therefore, it is a legitimate lower bound for the CB5 instance. The same is also true for CB13-CB14 instances, and CB29-CB30 instances.

The observations made for the Kartal data set such as decreasing number of cabinet-node links with the increasing values of $\gamma$, increasing coverage with increasing $p$ values for fixed $\gamma$ and $H$, sensitivity of the objective function value to the $p$ value and the QoS implications for different $\gamma$ and $p$ parameter values are 


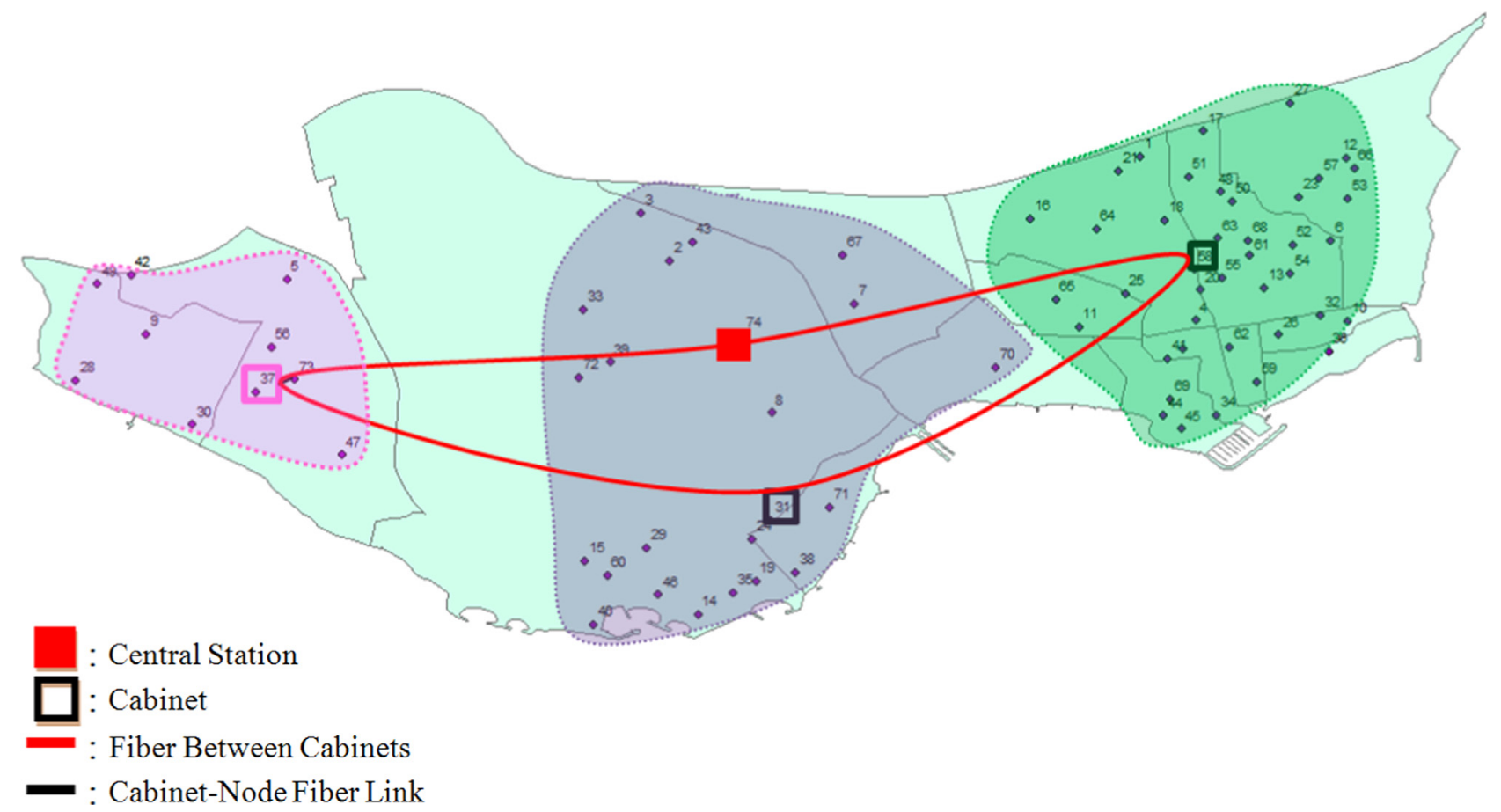

Fig. 18. Illustration of instance CB 2 .

Table 12

Copper field results for Bakırköy dataset.

\begin{tabular}{|c|c|c|c|c|c|c|c|}
\hline \multirow[t]{2}{*}{$\#$} & \multicolumn{3}{|c|}{ Parameters } & \multicolumn{4}{|l|}{ Results } \\
\hline & $\begin{array}{l}\gamma \\
(\mathrm{km})\end{array}$ & $p$ & $\begin{array}{l}H \\
(\mathrm{~km})\end{array}$ & $\mathrm{CPU}(\mathrm{s})$ & $\begin{array}{l}\text { Fiber cable } \\
\text { length } \\
\text { (Obj.Fn. } \\
\text { Val.) }(\mathrm{km})\end{array}$ & $\begin{array}{l}\text { Copper } \\
\text { cable } \\
\text { length } \\
(\mathrm{km})\end{array}$ & $\begin{array}{l}\text { Percentage } \\
\text { and (\#) of } \\
\text { nodes served } \\
\text { by fiber } \\
\text { cables }\end{array}$ \\
\hline CB1 & 0 & 3 & 15 & 1885.49 & 235.33 & 0.00 & $100.00 \%(74)$ \\
\hline $\mathrm{CB} 2$ & & & 20 & 49.61 & 143.45 & 0.00 & $100.00 \%(74)$ \\
\hline CB3 & & & 25 & 37.69 & 141.21 & 0.00 & $100.00 \%(74)$ \\
\hline CB4 & & & 50 & 48.04 & 141.21 & 0.00 & $100.00 \%$ \\
\hline CB5 & & 6 & 15 & Gap - 5.35\% & $(109.75)$ & 0.00 & $100.00 \%(74)$ \\
\hline CB6 & & & 20 & 7877.13 & 103.88 & 0.00 & $100.00 \%(74)$ \\
\hline CB7 & & & 25 & 4573.94 & 101.69 & 0.00 & $100.00 \%(74)$ \\
\hline CB8 & & & 50 & 320.34 & 97.96 & 0.00 & $100.00 \%(74)$ \\
\hline CB9 & 1 & 3 & 15 & 1371.65 & 233.27 & 3.01 & $94.59 \%(70)$ \\
\hline CB10 & & & 20 & 67.54 & 140.64 & 3.08 & $91.89 \%(68)$ \\
\hline CB11 & & & 25 & 59.71 & 137.31 & 4.69 & $87.84 \%$ (65) \\
\hline CB12 & & & 50 & 56.78 & 137.31 & 4.69 & $87.84 \%$ (65) \\
\hline CB13 & & 6 & 15 & Gap - 5.65\% & $(101.28)$ & 9.04 & $79.73 \%$ (59) \\
\hline CB14 & & & 20 & $15,057.88$ & 95.56 & 9.66 & $78.38 \%(58)$ \\
\hline CB15 & & & 25 & 3002.49 & 93.26 & 8.79 & $81.08 \%(60)$ \\
\hline CB16 & & & 50 & 1138.95 & 89.85 & 8.47 & 79.73\% (59) \\
\hline CB17 & 5 & 3 & 15 & $21,377.57$ & 215.69 & 35.65 & $81.08 \%(60)$ \\
\hline CB18 & & & 20 & 55.11 & 99.67 & 76.17 & $54.05 \%(40)$ \\
\hline CB19 & & & 25 & 58.69 & 98.87 & 86.61 & $54.05 \%(40)$ \\
\hline CB20 & & & 50 & 67.01 & 98.07 & 82.85 & $54.05 \%(40)$ \\
\hline CB21 & & 6 & 15 & $20,345.9$ & 43.86 & 147.37 & $20.27 \%(15)$ \\
\hline CB22 & & & 20 & 8716.32 & 42.24 & 147.37 & $20.27 \%(15)$ \\
\hline CB23 & & & 25 & 756.17 & 36.35 & 147.23 & $20.27 \%(15)$ \\
\hline CB24 & & & 50 & 1547.02 & 36.32 & 147.23 & $20.27 \%(15)$ \\
\hline CB25 & 10 & 3 & 15 & 8885.87 & 201.37 & 70.54 & $74.32 \%(55)$ \\
\hline CB26 & & & 20 & 70.43 & 74.32 & 140.03 & $47.30 \%(35)$ \\
\hline CB27 & & & 25 & 65.32 & 74.32 & 140.03 & $47.30 \%(35)$ \\
\hline CB28 & & & 50 & 61.32 & 74.32 & 140.03 & $47.30 \%(35)$ \\
\hline CB29 & & 6 & 15 & Gap - 19.74\% & $(29.08)$ & 232.65 & $9.46 \%(7)$ \\
\hline CB30 & & & 20 & $12,287.29$ & 23.34 & 232.00 & $9.46 \%(7)$ \\
\hline CB31 & & & 25 & $12,586.46$ & 23.25 & 228.86 & $9.46 \%(7)$ \\
\hline CB32 & & & 50 & $16,806.83$ & 23.25 & 228.86 & $9.46 \%(7)$ \\
\hline
\end{tabular}

valid for the Bakırköy data set as well. Therefore, for this data set, we discuss only some additional observations.

The objective function value depends on the $\gamma$ value, as expected. In Instance CB 1, there are 3 cabinets and the distance threshold is $15 \mathrm{~km}$ where none of the demand nodes is covered via the corresponding $\gamma$ value. For this instance, 70 cabinet-node links are needed. Cabinets are in points 8, 29, and 70. When we allow the $\gamma$ value to be $1 \mathrm{~km}$, in Instance CB 9, the locations of the cabinets are the same. However, this time there are only 66 cabinet-node links and the remaining 4 demand nodes are covered via a cabinet, as reported on the last column of the table.

Also, the number of cabinets has a strong effect on the structure of the networks. For example, as the cabinet number increases for the same $\gamma$ and $H$ values, the objective function value decreases. From Instance CB 19, where there are 3 cabinets to Instance CB 23 , where there are 6 cabinets, the objective function value decreases from $98.87 \mathrm{~km}$ to $36.35 \mathrm{~km}$. This difference is due to fewer cabinet-node link installations in the network.

Also, the same number of cabinets may be obtained for totally different parameter settings. In Instance CB 2, where the $\gamma$ value is 0 , the number of cabinets is 3 and the distance threshold is $20 \mathrm{~km}$, the cabinets are located at points 31, 37 and 58, as shown in Fig. 18.

In this figure, each cloud corresponds to a ring and every demand node in the cloud is connected to its cabinet in the cloud with a star topology. When we increase the $\gamma$ value to $1 \mathrm{~km}$, in Instance CB 10 , the same nodes are selected as cabinets. However, in Instance $C B$ 2, there are 70 cabinet-node links, whereas in Instance CB 10 , there are only 64 of them. Six nodes are covered via cabinets. This shows the effect of the $\gamma$ value.

\section{Conclusions}

This study introduces two new telecommunications network design problems arising from an application of the largest Internet service provider in Turkey to the literature. Mathematical models for the two problems, namely the green field network design 
problem and the copper field network re-design problem, are proposed. To test our mixed integer mathematical models, we perform computational analyses on data from two districts in Istanbul, Turkey, simulating real data. According to our computational analyses in the green field, the threshold Internet speed for each customer is a significant parameter that affects the total cost of setting up the network. As a managerial takeaway, increasing the Internet speed threshold in the green field network design problem causes fewer number of splitters being used, which in turn decreases the maximum $\mathrm{dB}$ values in the network. This is also a positive effect on the QoS. Thus increasing the threshold speed not only provides better service for the customers, but also decreases the optical losses in the network. This result is intact for different central node selections. On the other hand, the copper field experimental design shows the effect of the coverage distance, the number of cabinets and the distance threshold. As the coverage distance value and the number of cabinets increase, fewer number of cabinet-node links are needed in the final design.

Although the CPU performances vary a lot, extensive computational results show that the proposed models are viable exact solution methodologies for moderate dimensions necessitated by the company. Future work can be directed towards improved exact solution methodologies for these strategic level decisions.

\section{Acknowledgements}

We are thankful to anonymous referees for their constructive feedbacks in leading to the current form of this paper.

\section{References}

[1] Klincewicz JG. Hub location in backbone/tributary network design: a review. Location Science 1998;6:307-335.

[2] Ergun IY, Kırca O, Sural H. Network design problems in telecommunication. Technical report. Middle East Technical University, Ankara, Turkey; 2011.

[3] Gavish B. Topological design of telecommunication networks-local access design methods. Annals of Operations Research 1991;33:17-71.

[4] Gavish B, Li C, Simchi-levi D. Analysis of heuristics for the design of tree networks. Annals of Operations Research 1992;36:77-86.

[5] Balakrishnan A, Magnanti TL, Shulman A, Wong RT. Models for planning capacity expansion in local access telecommunication networks. Annals of Operations Research 1991;33:239-284.

[6] Chandy KM, Russell KM. The design of multipoint linkages in a teleprocessing tree network. IEEE Transactions on Communications 1972;21:1062-1066.

[7] Goldstein MC. Design of long-distance telecommunication networks-the Telepak problem. IEEE Transactions Circuit Theory 1973;20:186-192.

[8] Gourdin E, Labbe M, Yaman H. Telecommunication and location. In: Drezner Z, Hamacher HW, editors. Facility location. New York: Springer Berlin Heidelberg; 2002.

[9] Hansler E. A heuristic configuration procedure for cost minimal communication networks. Technical report. IBM Research Report RZ-666; 1974.

[10] Rothfarb B, Goldstein MC. The one-terminal Telepak problem. Operations Research 1971;19:156-169.

[11] Carpenter T, Luss H. Telecommunications access network design. In: In Resende MGC, Pardalos PM, editors. Handbook of optimization in telecommunications. US: Springer; 2006.

[12] Frantzeskakis LF, Luss $H$. The network redesign problem for access telecommunications networks. Naval Research Logistics 1999;46:488-506.

[13] Chamberland S. Global access network evolution. IEEE/ACM Transactions on Networking 2010;18:136-149.

[14] Randazzo CD, Luna HP. A comparison of optimal methods for local access uncapacitated network design. Annals of Operations Research 2001;106:263-286.

[15] Balakrishnan A, Magnanti TL, Wong RT. A decomposition algorithm for local access telecommunications network expansion planning. Operations Research 1995;43:58-76.

[16] Fischer M, Garbin D, Harris T, Knepley J. Large scale communications networks -design and analysis. Omega 1978;6:331-340.

[17] Altinkemer K, Yu Z. Topological design of wide area communication networks. Annals of Operations Research 1992;36:365-382.

[18] Gerla M, Kleinrock L. On the topological design of distributed computer networks. IEEE Transactions on Communications 1977;25:48-60.
[19] Kershenbaum A, Kermani P, Grover G. MENTOR: an algorithm for mesh network topological optimization and routing. IEEE Transactions on Communication 1991;39:503-513.

[20] Brandeau ML, Chiu SS. An overview of representative problems in location research. Management Science 1989;35:645-674.

[21] Daskin MS. Network and discrete location: models algorithms and applications. New York: Wiley/Interscience; 1995.

[22] Domschke W, Krispsin G. Location and layout planning: a survey. OR Spektrum 1997;19:181-194.

[23] Francis R, Mirchandani PB. Discrete location theory. New York: Wiley/Interscience; 1990.

[24] Francis R, White J. Facility layout and location: an analytic approach. Englewood Cliffs, NJ: Prentice-Hall; 1974.

[25] Handler GY, Mirchandani PB. Location on networks: theory and algorithms Cambridge, MA: MIT Press; 1979.

[26] Kershenbaum A. Telecommunication network design algorithms. New York: McGraw-Hill; 1993.

[27] Magnanti TL, Wong RT. Network design and transportation planning: models and algorithms. Transportation Science 1984;18:1-55.

[28] Tansel BC, Francis RL, Lowe TJ. Location on networks: a survey, Parts I and II Management Science 1983;29:482-511.

[29] Wong RT. Location and network design. In: O'hEigeartaigh M, Lenstra JK Rinnooy Kan AHG, editors. Combinatorial optimization: annotated bibliographies. New York: Wiley; 1985. p. 127-47.

[30] Effenbergera F, El-Bawabb TS. Passive optical networks (PONs): past present and future. Optical Switching and Networking 2009;6:143-150.

[31] Gutierrez D, Kim KS, Rotolo S, An F, Kazovsky LG. FTTH standards, deployments and research issues. In: Proceedings of the 8th joint conference on information sciences, Salt Lake City, UT, USA; 2005. p. 1358-61.

[32] Kim Y, Lee Y, Han J. A splitter location-allocation problem in designing fiber optic access networks. European Journal of Operational Research 2011;210:425-435.

[33] Brittain D, Williams S, Hale P, Brewer D, Asumu D. External network planningcomparing and contrasting optimization tools across various deployment scenarios. In: European conference on networks and optical communications; 1997. p. $218-23$

[34] Chardy M, Costa MC, Faye A, Trampont M. Optimizing splitter and fiber location in a multilevel optical FTTH network. European Journal of Operational Research 2012;222:430-440.

[35] Lv M, Chen X. Heuristic based multi-hierarchy passive optical network planning. In: Wireless communications, networking and mobile computing, 2009 WiCom'09. 5th International Conference; 2009.

[36] Kokangul A, Ari A. Optimization of passive optical network planning. Applied Mathematical Modelling 2011;35:3345-3354.

[37] Minoux M. Network synthesis and optimum network design problems: models, solution methods and applications. Networks 1989;19:313-360.

[38] Basharin G, Rusina N. Multi-rate loss model for optical network unit in passive optical networks. In: Distributed computer and communication networks. Springer International Publishing, AG, Cham; 2014. p. 219-28.

[39] Andreas B, Ljubić I, Maurer O. Lagrangian decompositions for the two-leve FTTx network design problem. EURO Journal on Computational Optimization 2013;1(3-4):221-252.

[40] Contreras I, Fernández E, Marín A. The tree of hubs location problem. European Journal of Operational Research 2010:202(2):390-400.

[41] Contreras I, Fernández E, Marín A. Tight bounds from a path based formulation for the tree of hub location problem. Computers \& Operations Research 2009;36(12):3117-3127.

[42] de Sá EM, de Camargo RS, de Miranda G. An improved Benders decomposition algorithm for the tree of hubs location problem. European Journal of Operational Research 2013;226(2):185-202.

[43] Park C, Lee Y, Kim Y, Park G. An access network design problem with end-toend QoS constraints. Omega 2014;48:36-48.

[44] Park G, Lee Y, Han J. A two-level location-allocation problem in designing local access fiber optic networks. Computers \& Operations Research 2014;51:52-63.

[45] Costa AM, França PM, Lyra Filho C. Two-level network design with intermediate facilities: an application to electrical distribution systems. Omega 2011;39(1):3-13.

[46] Doubleday CF, Probert DE, Walsham G. A strategic model of communications demand. Omega 1983;11(4):343-354.

[47] Leea D-J, Ahnb J-K. Factors affecting companies' telecommunication service selection strategy. Omega 2007;35:486-493.

[48] Ljubić I, Putz P, Salazar-Gonzalez J-J. A MIP-based approach to solve the prizecollecting local access network design problem. European Journal of Operational Research 2014;235(3):727-739.

[49] Premkumar G. Strategic telecommunications planning-a descriptive model Omega 1990;18(5):447-462.

[50] Li Ji, Gangxiang Shen. Cost minimization planning for passive optical networks. In: National Fiber Optic Engineers Conference, Washington, DC, USA 2008, pp. 1-3.

[51] Kilci F, Kara BY. Locating temporary shelter areas after an earthquake: a case for Turkey. European Journal of Operational Research 2015;243(1):323-332.

[52] Miller, Clair E, Albert W Tucker, Richard A Zemlin. Integer programming formulation of traveling salesman problems. Journal of the ACM 1960;7(4):326-9.

[53] Tarjan Robert E. Sensitivity analysis of minimum spanning trees and shortest path trees. Information Processing Letters 1982;14(1):30-33. 